\title{
Teichmüller space via Kuranishi families
}

\author{
ENRICO ARBARELlo AND MAURIZIO CORnALBA
}

\begin{abstract}
In this partly expository note we construct Teichmüller space by patching together Kuranishi families. We also discuss the basic properties of Teichmüller space, and in particular show that our construction leads to simplifications in the proof of Teichmüller's theorem asserting that the genus $g$ Teichmüller space is homeomorphic to a $(6 g-6)$-dimensional ball.
\end{abstract}

Mathematics Subject Classification (2000): 30F60 (primary); 14H15, 32G15, 14H10 (secondary).

\section{Introduction}

Our main goal, in this partly expository note, is to show how the genus $g$ Teichmüller space $T_{g}$ and the universal family over it can be constructed by patching together Kuranishi families of genus $g$ curves. This approach, which is close in spirit to the one of Grothendieck [20,21], should appeal in particular to readers with an algebro-geometric background, as it relies mostly on standard tools and methods of their trade. In fact, the main objects we use are the Kuranishi families of smooth genus $g$ curves, whose construction and properties can be derived quite directly from the theory of the Hilbert scheme. Our point of view seems to have several advantages over more traditional ones. First of all, Teichmüller space is constructed directly as a complex manifold, and the construction makes it obvious that it enjoys a natural universal property. Secondly, it is very easy to show that the action of the Teichmüller modular group on $T_{g}$ is properly discontinuous. Finally, our presentation provides a shortcut to the proof of Teichmüller's theorem, which states that $T_{g}$ is homeomorphic to a $(6 g-6)$-dimensional ball $B_{g}$. In fact, we show that the universal property of the Kuranishi family holds not only with respect to analytic deformations, but also with respect to continuous ones, and observe that this immediately proves that the Teichmüller map $\Phi: B_{g} \rightarrow T_{g}$ is continuous. This first step eliminates some of the technicalities involved in the usual proof of Teichmüller's

Research partially supported by: PRIN 2005 Spazi di moduli e teoria di Lie; FAR 2004 (Pavia) Varietà algebriche, calcolo algebrico, grafi orientati e topologici.

Received February 18, 2008; accepted July 9, 2008. 
theorem, highlighting the two central points of the argument: Teichmüller's uniqueness theorem and the existence of solutions of the Beltrami equation. It should be observed that the same program can be carried out for the Teichmüller space $T_{g, n}$ of genus $g$ curves with $n$ marked points, although this will not be done here. In the bibliography we have provided references to the most fundamental texts in this very classical theory.

\section{The Kuranishi family}

Throughout this section, $C$ will denote a compact connected Riemann surface of genus $g>1$, or more briefly, a curve of genus $g>1$. A family of genus $g$ curves parametrized by an analytic space $S$ is a proper surjective analytic map $\eta: X \rightarrow S$ having genus $g$ compact Riemann surfaces as fibers. If $s$ is a point of $S$, we shall write $X_{s}$ to indicate the fiber $\eta^{-1}(s)$; more generally, we shall use the convention of appending the subscript $s$ to an object over $S$ to indicate its "fiber" at $s$. The precise meaning of this in each case will be clear from the context. We will say that $\eta: X \rightarrow S$ is a family of genus $g$ curves in $\mathbb{P}^{N}$, parametrized by $S$, if $X$ is a subvariety of $\mathbb{P}^{N} \times S$ and $\eta$ is the restriction to $X$ of the projection on the second factor. A deformation of $C$, parametrized by a pointed analytic space $\left(S, s_{0}\right)$, is a family of genus $g$ curves $\eta: X \rightarrow S$, together with an isomorphism $\psi: C \stackrel{\simeq}{\rightarrow} X_{s_{0}}$. Thus, a deformation of $C$ is determined by the data $(\eta, \psi)$. Given a neighborhood $U$ of $s_{0}$, we let $\eta_{U}: \eta^{-1}(U) \rightarrow U$ be the map obtained by restriction from $\eta$. If $\eta^{\prime}: X^{\prime} \rightarrow S^{\prime}, \psi^{\prime}: C \rightarrow X_{s_{0}^{\prime}}^{\prime}$ is another deformation of $C$, parametrized by a pointed analytic space $\left(S^{\prime}, s_{0}^{\prime}\right)$, a morphism of deformations from $(\eta, \psi)$ to $\left(\eta^{\prime}, \psi^{\prime}\right)$ is a pair $(f, F)$ of morphism fitting in a Cartesian diagram

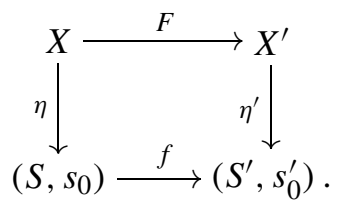

Let $\left(B, b_{0}\right)$ be a $(3 g-3)$-dimensional connected pointed complex manifold. A Kuranishi family for $C$ is a deformation of $C$ parametrized by $\left(B, b_{0}\right)$ :

$$
\pi: \mathcal{C} \rightarrow B, \quad \varphi: C \stackrel{\simeq}{\rightarrow} \mathcal{C}_{b_{0}}
$$

satisfying the following universal property. Given a deformation $(\eta, \psi)$ of $C$ as above, there are a neighborhood $U$ of $s_{0}$ and a morphism $(f, F)$ of deformations from $\left(\eta_{U}, \psi\right)$ to $(\pi, \varphi)$; moreover, this morphism is essentially unique, in the sense that any other morphism of this sort agrees with $(f, F)$ over $\eta^{-1}\left(U^{\prime}\right) \rightarrow U^{\prime}$, for some neighborhood $U^{\prime}$ of $s_{0}$.

Kuranishi families for curves of genus $g>1$ always exist. We will now briefly illustrate how one such family may be constructed. Let $v \geq 3$ and set 
$N=(2 v-1)(g-1)-1$. As is well known, a curve of genus $g$ can be embedded in the projective space $\mathbb{P}^{N}$ via the $v$-canonical map, that is, via the sections of the $\nu$-th power of the canonical line bundle $\omega_{C}$. The embedding depends on the choice of a basis for the space of sections of $\omega_{C}^{v}$, and different choices of basis give rise to projectively equivalent embeddings. Conversely, any isomorphism between two genus $g$ curves comes from a unique projectivity of $\mathbb{P}^{N}$ carrying the $v$-canonical image of one curve to the $v$-canonical image of the other. The Hilbert scheme $H_{g, v}$ of $v$-canonical, genus $g$ curves is a smooth projective variety whose points are in a one-to-one correspondence with the set of $\nu$-canonically embedded curves of genus $g$. The Hilbert scheme $H_{g, v}$ is the parameter space for a family $\xi: \mathcal{Y} \rightarrow H_{g, v}$ of genus $g$ curves in $\mathbb{P}^{N}$ having the following universal property. For every family $\eta: X \rightarrow S$ of genus $g$ curves $\nu$-canonically embedded in $\mathbb{P}^{N}$ there exists a unique morphism $\alpha: S \rightarrow H_{g, v}$ exhibiting the family $\eta$ as the pull-back, via $\alpha$, of the universal family $\xi$, so that there is a commutative diagram of Cartesian squares

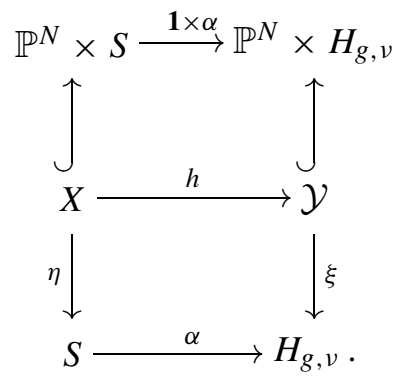

The projective group $P G L(N+1, \mathbb{C})$ acts naturally on $H_{g, v}$, with finite stabilizers, since curves of genus $g>1$ have finite automorphism groups. Thus, in accordance with Riemann's count,

$$
\operatorname{dim} H_{g, v}=3 g-3+\operatorname{dim} P G L(N+1, \mathbb{C}) .
$$

Now let us consider a genus $g$ curve $C$ and a $\nu$-canonical embedding $\varphi: C \stackrel{\simeq}{\rightarrow}$ $\Gamma \subset \mathbb{P}^{N}$ of $C$, associated to a basis $\chi_{0}, \ldots, \chi_{N}$ of the space of $v$-fold holomorphic differentials on $C$. Let $p \in H_{g, v}$ be the point corresponding to $\Gamma$. We choose a local "slice" at $p$ for the action of $P G L(N+1, \mathbb{C})$, that is, a $(3 g-3)$-dimensional locally closed subvariety $U$ passing through $p$ and transverse to the orbits of $P G L(N+1, \mathbb{C})$. Possibly after shrinking $U$, if $W$ is a sufficiently small open neighborhood of the identity in the projective group, $(g, u) \mapsto g u$ is an isomorphism between $W \times U$ and an open neighborhood $V$ of $p$. Thus there is a canonical fibration $\sigma: V \rightarrow U$ along the orbits of $\operatorname{PGL}(N+1, \mathbb{C})$. Let now $\pi: \mathcal{C} \rightarrow U$ be the restriction to $U$ of the universal family $\xi: \mathcal{Y} \rightarrow H_{g, v}$. This family, together with the identification $\varphi: C \rightarrow \Gamma=\pi^{-1}(p)$, is a Kuranishi family for $C$. Let us see why. Suppose

$$
\eta: X \rightarrow S, \quad \psi: C \rightarrow X_{s_{0}}
$$


is a deformation of $C$ parametrized by $\left(S, s_{0}\right)$. Consider the sheaf $\omega_{\eta}$ of relative holomorphic differentials along the fibers of $\eta$. By suitably shrinking the parameter space $S$ of this deformation we may assume that $\eta_{*} \omega_{\eta}^{v}$ is trivial on $S$. Choosing a frame of this vector bundle exhibits $\eta: X \rightarrow\left(S, s_{0}\right)$ as a family of $\nu$-canonically embedded curves, that is, determines an embedding $\beta: X \rightarrow \mathbb{P}^{N} \times S$ whose fiber $\beta_{s}: X_{s} \rightarrow \mathbb{P}^{N}$ at $s$ is $v$-canonical for every $s \in S$. Furthermore, if the frame is chosen so as to pull back to $\chi_{0}, \ldots, \chi_{N}$ on $C$, then the composition of the identification $\psi: C \rightarrow X_{s_{0}}$ with $\beta_{s_{0}}: X_{s_{0}} \rightarrow \mathbb{P}^{N}$ is equal to the composition of $\varphi$ with the inclusion of $\Gamma$ in $\mathbb{P}^{N}$. The universal property of the Hilbert scheme then yields a morphism $\alpha: S \rightarrow H_{g, v}$ such that $\alpha\left(s_{0}\right)=p$. We may assume that $\alpha(S) \subset V$. It is then immediate to verify that the morphism $\sigma \alpha: S \rightarrow U$ realizes the universal property of a Kuranishi family.

Possibly shrinking $B$, one can assume that the Kuranishi family (2.1) satisfies various useful additional properties. For instance, one can set things up so that (2.1) is "Kuranishi at every point of $B$ ". This means that, for any $b \in B$, the deformation consisting of $\mathcal{C} \rightarrow B$ and of the identity isomorphism from $\mathcal{C}_{b}$ to itself is a Kuranishi family for $\mathcal{C}_{b}$. In fact, the Kuranishi family whose construction we outlined above has this property.

We may also assume that the automorphism group of $C$ acts on $\mathcal{C}$ and $B$. In fact, let $\gamma$ be an automorphism of $C$. Then

$$
\pi: \mathcal{C} \rightarrow B, \quad \varphi \gamma: C \stackrel{\simeq}{\rightarrow} \mathcal{C}_{b_{0}}
$$

is another Kuranishi family. By the universal property, up to shrinking $B$, if necessary, there is a unique Cartesian diagram

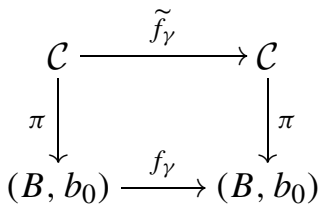

where $f_{\gamma}$ and $\tilde{f}_{\gamma}$ are isomorphisms, inducing an isomorphism between the two deformations of $C$ given by (2.1) and (2.2). This results, possibly after further shrinking of $B$, in compatible actions of $\operatorname{Aut}(C)$ on $B$ and $\mathcal{C}$ pulling back, via $\varphi$, to the standard action on $C$. The action on $\mathcal{C}$ is always faithful, while the action on $B$ is not faithful only when $g=2$; in this case the only elements of $\operatorname{Aut}(C)$ acting trivially are the identity and the hyperelliptic involution. By further shrinking the base $B$ one may even assume that any isomorphism between two fibers $\mathcal{C}_{b}$ and $\mathcal{C}_{b^{\prime}}$ of $\pi$ comes by restriction from $\widetilde{f}_{\gamma}$, for some $\gamma \in \operatorname{Aut}(C)$. This is often crucial in applications.

We end this section by recalling a fundamental compactness property of families of compact Riemann surfaces. Suppose $\alpha: X \rightarrow U$ and $\alpha^{\prime}: X^{\prime} \rightarrow U^{\prime}$ are two families of compact Riemann surfaces of genus $g>1$ parametrized by analytic spaces $U$ and $U^{\prime}$. Let $\left\{u_{n}\right\}_{n \in \mathbb{N}}\left(\left\{u_{n}^{\prime}\right\}_{n \in \mathbb{N}}\right)$ be a sequence of points in $U$ 
(respectively, $U^{\prime}$ ) converging to a point $u \in U$ (respectively, $u^{\prime} \in U^{\prime}$ ). Assume that $X_{u_{n}}$ is isomorphic to $X_{u_{n}^{\prime}}^{\prime}$, for every $n$. Then $X_{u}$ is isomorphic to $X_{u^{\prime}}^{\prime}$. This can be deduced from the following purely algebro-geometric statement, which is in turn a rather straightforward consequence of the properness of relative Hilbert schemes and of the theory of minimal models of algebraic surfaces. Let $Y \rightarrow R$ and $Y^{\prime} \rightarrow R$ be algebraic families of smooth curves of genus $g>1$; then the scheme $\operatorname{Isom}_{R}\left(Y, Y^{\prime}\right)$ parametrizing isomorphisms $Y_{r} \stackrel{\simeq}{\rightarrow} Y_{r}^{\prime}, r \in R$, is proper over $R$. Here is how the reduction to this statement of the preceding one goes. Let $Z \rightarrow S$ and $W \rightarrow T$ be algebraic families of smooth curves of genus $g>1$. Set $Y=Z \times T$, $Y^{\prime}=S \times W, R=S \times T$. Then the locus $\left\{(s, t): X_{S}\right.$ is isomorphic to $\left.Y_{t}\right\} \subset S \times T=R$ is the projection of $\operatorname{Isom}_{R}\left(Y, Y^{\prime}\right)$, and hence is closed. This shows in particular that the statement to be proved is true for algebraic families. To prove it in general it then suffices to show that the original families $\alpha: X \rightarrow U$ and $\alpha^{\prime}: X^{\prime} \rightarrow U^{\prime}$, or just their restrictions to suitable neighborhoods of $u$ and $u^{\prime}$, come by pullback from algebraic families. But this is clear. In fact, up to shrinking $U$ and $U^{\prime}$, we may suppose that $\alpha_{*} \omega_{\alpha}^{v}$ and $\alpha_{*}^{\prime} \omega_{\alpha^{\prime}}^{v}$ are trivial for some $v \geq 3$. Thus we may embed $X$ and $X^{\prime} v$-canonically, so $X \subset \mathbb{P}^{N} \times U, X^{\prime} \subset \mathbb{P}^{N} \times U^{\prime}$, and both families come from the universal family over the Hilbert scheme $H_{g, v}$ via maps $U \rightarrow H_{g, v}$ and $U^{\prime} \rightarrow H_{g, v}$.

A convenient reference for deformation theory and Hilbert schemes is [33]. A detailed presentation of Kuranishi families will be contained in the forthcoming volume [6].

\section{The construction of Teichmüller space as a complex manifold}

Fix an oriented genus $g$ topological surface $\Sigma$. We assume that $g>1$. Given a genus $g$ curve $C$, a Teichmüller structure on it is the isotopy class $[f]$ of an orientation-preserving homeomorphism $f: C \rightarrow \Sigma$. An isomorphism between curves with Teichmüller structure $(C,[f])$ and $\left(C^{\prime},\left[f^{\prime}\right]\right)$ is an isomorphism of curves $\varphi: C \rightarrow C^{\prime}$, such that $\left[f^{\prime} \varphi\right]=[f]$. The set of isomorphism classes of genus $g$ curves with Teichmüller structure has a natural topology and complex structure which we are presently going to describe. The resulting space is called the Teichmüller space of $\Sigma$ and is denoted by the symbol $T_{\Sigma}$. The point in $T_{\Sigma}$ associated to the curve $C$ and to the isotopy class $[f]$ will be denoted by the symbol $[C,[f]]$. If $\Sigma^{\prime}$ is another oriented genus $g$ surface, and $\rho: \Sigma \rightarrow \Sigma^{\prime}$ an oriented homeomorphism, we get a bijection $T_{\Sigma} \rightarrow T_{\Sigma^{\prime}}$ by sending $[C,[f]]$ to $[C,[\rho \circ f]]$. Clearly, this bijection depends only on the isotopy class of $\rho$, and it will be clear from the construction of the complex structure on Teichmüller space that it is an isomorphism of complex manifolds. We are therefore justified in writing $T_{g}$, instead of $T_{\Sigma}$, when the reference surface $\Sigma$ is kept fixed, as will usually happen.

It is important to remark that a curve with Teichmüller structure $(C,[f])$ has no non-trivial automorphism. In fact, an automorphism $\varphi: C \rightarrow C$ such that $[f \varphi]=[f]$ must be homotopically trivial and, in particular, must induce the iden- 
tity on complex cohomology. This implies that $\varphi$ induces the identity at the level of holomorphic forms, and hence must commute with the canonical map. This shows that $f$ is the identity if $C$ is not hyperelliptic, and that $f$ is the identity or the hyperelliptic involution otherwise. However, the hyperelliptic involution does not act trivially on complex cohomology, so $f$ must be the identity in this case as well.

We next introduce a topology and a complex structure on Teichmüller space. We begin by extending the notion of Teichmüller structure from curves to families of curves. A Teichmüller structure on a family of genus $g$ curves $\eta: X \rightarrow S$ is the datum of a Teichmüller structure $\left[f_{s}\right]$ on each fiber $X_{s}$, satisfying the following coherence condition. There exists a cover of $S$ with open sets $U$ together with topological trivializations $\left(F, \eta_{U}\right): \eta^{-1}(U) \stackrel{\simeq}{\rightarrow} \Sigma \times U$ such that, for each $s \in U$, $\left[F_{S}\right]=\left[f_{S}\right]$. Let $V$ be an open subset of $S$, and let

$$
\begin{aligned}
& \left(F, \eta_{V}\right): \eta^{-1}(V) \stackrel{\simeq}{\rightarrow} \Sigma \times V \\
& \left(G, \eta_{V}\right): \eta^{-1}(V) \stackrel{\simeq}{\rightarrow} \Sigma \times V
\end{aligned}
$$

be two topological trivializations. Suppose that $\left[F_{s}\right]=\left[G_{s}\right]$ for some $s \in V$. Let $\gamma:[0,1] \rightarrow V$ be a path from a point $s^{\prime} \in V$ to $s$. Then, as $t$ varies between 0 and 1 , the homeomorphisms

$$
G_{\gamma(t)} \circ F_{\gamma(t)}^{-1} \circ F_{s^{\prime}}: X_{s^{\prime}} \rightarrow \Sigma
$$

describe an isotopy between $G_{s^{\prime}}$ and $G_{s} \circ F_{s}^{-1} \circ F_{s^{\prime}}$, which in turn is isotopic to $F_{s^{\prime}}$. Hence $\left[F_{s^{\prime}}\right]=\left[G_{s^{\prime}}\right]$ for all $s^{\prime} \in V$ belonging to the same connected component as $s$. This implies in particular that, when $V$ is connected, $F$ and $G$ are equal if and only if they agree at one point.

Now let $(C,[f])$ be a genus $g$ curve with Teichmüller structure. Consider a Kuranishi family (2.1) for the curve $C$. Possibly after shrinking $B$, such a family admits a topological trivialization $(F, \pi): \mathcal{C} \rightarrow \Sigma \times B$ such that $F_{b_{0}} \circ \varphi=f$, and hence can be endowed with a unique Teichmüller structure extending the one on $C$. We thus get what we shall refer to as a Kuranishi family for the curve with Teichmüller structure $(C,[f])$. The name is justified by the fact that such a family enjoys, with respect to deformations of curves with Teichmüller structure, a universal property exactly analogous to the one of standard Kuranishi families, as follows immediately from the universal property of ordinary Kuranishi families and the uniqueness of the Teichmüller structure extending the one on the fiber at $s_{0}$. It should be observed that, when $B$ is small enough, the family we just constructed is Kuranishi at every point of $B$, as follows from the analogous property of standard Kuranishi families.

We are ready to describe the topology and the complex structure on $T_{g}$. Let $y=[C,[f]]$ be a point of $T_{g}$. Choose a Kuranishi family for $(C,[f])$. As we explained, this can be constructed by putting on the family (2.1), where $B$ is chosen to be connected and "sufficiently small", the unique Teichmüller structure extending $[f]$; for each $b \in B$, we denote by $\left[F_{b}\right]$ the Teichmüller structure on $C_{b}$. As we 
pointed out at the end of Section 2, we may suppose that the action of $G=\operatorname{Aut}(C)$ on $C$ extends to an equivariant action on $\mathcal{C}$ and on $B$, and that any isomorphisms between fibers of $\pi$ is the restriction of the action of an element of $G$ on $\mathcal{C}$. We claim that the natural map

$$
\begin{aligned}
\alpha: B & \longrightarrow T_{g} \\
b & \mapsto\left[C_{b},\left[F_{b}\right]\right]
\end{aligned}
$$

is injective. A map of this sort will be called a standard coordinate patch for $T_{g}$ around the point $y$. To prove the injectivity of $\alpha$, suppose that $\alpha(b)=\alpha\left(b^{\prime}\right)$ for $b, b^{\prime} \in B$. Thus there is an isomorphism $\psi: C_{b} \rightarrow C_{b^{\prime}}$ such that $\left[F_{b}\right]=\left[F_{b^{\prime}} \circ \psi\right]$. Our assumptions imply that $\psi$ is induced by an element $\rho \in G$, so that there is a commutative diagram

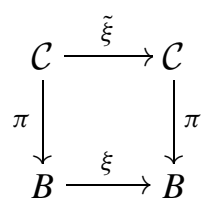

where $\tilde{\xi}$ and $\xi$ are automorphisms such that $\xi\left(b_{0}\right)=b_{0}, \xi(b)=b^{\prime}, \tilde{\xi}_{b}=\psi$, and $\tilde{\xi}$ pulls back to $\rho$ on $C$. We define a new Teichmüller structure on (2.1) by setting $F_{t}^{\prime}=F_{\xi(t)} \circ \tilde{\xi}_{t}$. Since $\left[F_{b}^{\prime}\right]=\left[F_{b^{\prime}} \circ \psi\right]=\left[F_{b}\right]$, it follows that $\left[F_{t}^{\prime}\right]=\left[F_{t}\right]$ for every $t \in B$. When $t=b_{0}$, this says that $[f \circ \rho]=[f]$. Thus $\rho$ is isotopic to the identity and, as we observed above, this implies that $\rho=1$, proving our claim.

Let us show that the patches we have just introduced define a complex structure on $T_{g}$. Let $\alpha: B \rightarrow T_{g}$ and $\beta: B^{\prime} \rightarrow T_{g}$ be two standard patches whose codomains have a point $z$ in common. Let $\pi: \mathcal{C} \rightarrow B, \pi^{\prime}: \mathcal{C}^{\prime} \rightarrow B^{\prime}$ be the corresponding families, and $U$ a small enough neighborhood of $\beta^{-1}(z)$. Then, by the universal property of Kuranishi families, there is a unique morphism of families of curves with Teichmüller structure

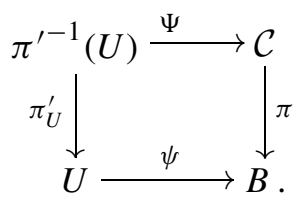

On the other hand, $\psi$ clearly agrees with the restriction to $U$ of $\alpha^{-1} \circ \beta$. In particular, $\beta^{-1}(\alpha(B))$ is open in $B^{\prime}$, and $\alpha^{-1} \circ \beta$ is holomorphic on it.

This completes the construction of a (possibly non-Hausdorff) complex structure on $T_{g}$; in the next section we shall prove that $T_{g}$ is actually a Hausdorff topological space. In fact, the above argument also shows that the Kuranishi families corresponding to standard coordinate patches can be canonically glued together to yield a universal family of curves with Teichmüller structure

$$
\eta: \mathcal{X}_{g} \longrightarrow T_{g}
$$


This family is universal in the sense that any holomorphic family of genus $g$ curves with Teichmüller structure over a base $T$ is isomorphic, via a unique isomorphism, to the pull-back of the family $\mathcal{X}_{g} \rightarrow T_{g}$ via a unique morphism $T \rightarrow T_{g}$. In particular, $T_{g}$ represents the functor

$$
T \mapsto\left\{\begin{array}{c}
\text { isomorphism classes of holomorphic families of } \\
\text { genus } g \text { curves with Teichmüller structure over } T
\end{array}\right\}
$$

from analytic spaces to sets.

\section{The mapping class group and its action}

As in the previous section, we pick a reference oriented genus $g$ topological surface $\Sigma$. The mapping class group $\Gamma_{\Sigma}$, also called Teichmüller modular group, is the group of all isotopy classes of orientation-preserving homeomorphism of $\Sigma$. When the reference surface $\Sigma$ is kept fixed, we shall often denote it by $\Gamma_{g}$. The mapping class group acts naturally on $T_{g}=T_{\Sigma}$, the action of an element $[\gamma]$ being given by

$$
[\gamma] \cdot[C,[f]]=[C,[\gamma \circ f]] .
$$

It is immediate to show that the elements of $\Gamma_{g}$ act on $T_{g}$ as holomorphic automorphisms. Consider an element $[\gamma] \in \Gamma_{g}$, and a standard coordinate patch $\alpha: B \rightarrow T_{g}$ obtained from a Kuranishi family (2.1), endowed with a Teichmüller structure $\left\{F_{b}\right\}_{b \in B}$. We know that $[\gamma]$ acts by replacing each Teichmüller structure $[f]$ with $[\gamma \circ f]$. Substituting $\left\{\gamma \circ F_{b}\right\}_{b \in B}$ for the Teichmüller structure $\left\{F_{b}\right\}_{b \in B}$ produces a new coordinate patch $\beta: B \rightarrow T_{g}$, and it is clear that, for any $b \in B$,

$$
\beta^{-1}([\gamma] \cdot \alpha(b))=b .
$$

This shows that $[\gamma]$ acts holomorphically.

As a set, the moduli space $M_{g}$ of genus $g$ curves is the set of isomorphism classes of smooth curves of genus $g$. Ignoring Teichmüller structures gives a map

$$
m: T_{g} \rightarrow M_{g}
$$

which can be identified with the quotient map from $T_{g}$ to $T_{g} / \Gamma_{g}$. To see this first observe that, given $y \in T_{g}$ and $[\gamma] \in \Gamma_{g}, y$ and $[\gamma] \cdot y$ obviously map to the same point of $M_{g}$. Conversely, given points $y=[C,[f]]$ and $y^{\prime}=\left[C^{\prime},\left[f^{\prime}\right]\right]$ of $T_{g}$ mapping to the same point of $M_{g}$, there is an isomorphism $\varphi: C \rightarrow C^{\prime}$, so that $y^{\prime}=[\gamma] \cdot y$, where $\gamma=f^{\prime} \varphi f^{-1}$. Let now $G$ be the automorphism group of the curve $C$. We claim that the homomorphism $\sigma$ from $G$ to $\Gamma_{g}$ given by

$$
\rho \mapsto\left[\gamma_{\rho}\right] \text {, where } \gamma_{\rho}=f \rho f^{-1},
$$

identifies $G$ with the stabilizer of $y$. First of all, $\sigma$ is injective, since $\left[f \rho f^{-1}\right]=1$ if and only if $\rho$ is homotopic to the identity, and, as we know, this happens only 
when $\rho=1$. Furthermore, $f \rho=\gamma_{\rho} f$, which exactly means that $\rho$ is an isomorphism between the curves with Teichmüller structure $(C,[f])$ and $\left(C,\left[\gamma_{\rho} f\right]\right)$. Thus $y=\left[\gamma_{\rho}\right] \cdot y$, that is, $\sigma(\rho)$ belongs to the stabilizer of $y$. Finally, given any $[\gamma]$ in the stabilizer of $y$, there is an element $\psi$ of $G$ such that $[f \psi]=[\gamma f]$, that is, $[\gamma]=\left[\gamma_{\psi}\right]=\sigma(\psi)$ for some $\psi \in G$.

Now consider a standard coordinate patch $\alpha: B \rightarrow T_{g}$ centered at $y$, coming from a Kuranishi family (2.1), endowed with a Teichmüller structure $\left\{F_{b}\right\}_{b \in B}$. We shall prove that $\alpha$ is $G$-equivariant. If $\rho$ is an element of $G$, there is a diagram (3.1), where $\tilde{\xi}$ and $\xi$ are automorphisms such that $\xi\left(b_{0}\right)=b_{0}$ and $\tilde{\xi}_{b_{0}} \varphi=\varphi \rho$. The action of $\rho$ on $B$ is then given by $\rho \cdot b=\xi(b)$. We must show that $\alpha(\xi(b))=[\gamma] \cdot \alpha(b)$, where $\gamma=\gamma_{\rho}=f \rho f^{-1}$. We define two new Teichmüller structures $\left\{H_{b}\right\}_{b \in B}$ and $\left\{K_{b}\right\}_{b \in B}$ on (2.1) by setting $H_{b}=F_{\xi(b)} \tilde{\xi}_{b}$ and $K_{b}=\gamma F_{b}$. It is easy to verify that $H_{b_{0}}=K_{b_{0}}$. It follows that $\left[H_{b}\right]=\left[K_{b}\right]$ for all $b$. Since $\tilde{\xi}_{b}$ is an isomorphism between $\left(C_{b},\left[H_{b}\right]\right)$ and $\left(C_{\xi(b)},\left[F_{\xi(b)}\right]\right)$, and since

$$
[\gamma] \cdot\left[C_{b},\left[F_{b}\right]\right]=\left[C_{b},\left[K_{b}\right]\right]
$$

we have that

$$
\left[\gamma_{\rho}\right] \cdot \alpha(b)=\left[C_{b},\left[K_{b}\right]\right]=\left[C_{b},\left[H_{b}\right]\right]=\alpha(\xi(b))
$$

as wished.

The moduli space $M_{g}=T_{g} / \Gamma_{g}$ is equipped with the quotient topology. A basis for this topology is given as follows. As we just saw, any standard patch $\alpha: B \rightarrow T_{g}$ drops to an injective map $\bar{\alpha}: B / G \rightarrow M_{g}$, where $G$ is the automorphism group of the central fiber. The open sets of the form $\bar{\alpha}(B / G)$ form a basis for the topology of $M_{g}$.

The fact that the moduli space $M_{g}$ is Hausdorff is an immediate consequence of the property mentioned at the end of Section 2. Suppose in fact that the sequence $\left\{z_{n}\right\} \subset M_{g}$ converges to two points $x$ and $y$, corresponding to the isomorphism classes of curves $C$ and $C^{\prime}$. Consider Kuranishi families $\pi: \mathcal{C} \rightarrow B$ and $\pi^{\prime}: \mathcal{C}^{\prime} \rightarrow B^{\prime}$ for $C=\pi^{-1}\left(b_{0}\right)$ and $C^{\prime}=\pi^{-1}\left(b_{0}^{\prime}\right)$, respectively, yielding standard patches $\alpha: B \rightarrow T_{g}$ and $\alpha^{\prime}: B^{\prime} \rightarrow T_{g}$. We may assume that $\left\{z_{n}\right\} \subset \bar{\alpha}(B) \cap \bar{\alpha}^{\prime}\left(B^{\prime}\right)$. Lift the sequence $\left\{z_{n}\right\}$, via $\bar{\alpha}$ and $\bar{\alpha}^{\prime}$, to sequences $\left\{x_{n}\right\} \subset B$ and $\left\{y_{n}\right\} \subset B^{\prime}$ converging, respectively, to $b_{0}$ and $b_{0}^{\prime}$. Since $C_{x_{n}}$ is isomorphic to $C_{y_{n}}^{\prime}$, for all $n$, we conclude that $C \cong C_{b_{0}}$ is isomorphic to $C^{\prime} \cong C_{b_{0}^{\prime}}^{\prime}$, so that $x=y$.

We shall now show that $T_{g}$ is Hausdorff and, at the same time, that the action of $\Gamma_{g}$ on $T_{g}$ is properly discontinuous. We must prove that:

1) Points $y$ and $y^{\prime}$ in $T_{g}$ belonging to different $\Gamma_{g}$-orbits possess disjoint neighborhoods.

2) Every point $y \in T_{g}$ possesses a neighborhood $V$ such that, if $G_{y}$ is the stabilizer of $y$, then

$$
\left\{\gamma \in \Gamma_{g}: \gamma V \cap V \neq \emptyset\right\} \subset G_{y} .
$$


The first property is a direct consequence of the Hausdorffness of $M_{g}$. To prove the second, fix $y \in T_{g}$. We can then identify the stabilizer $G_{y}$ with the automorphism group of the fiber $\mathcal{X}_{y}$ of the universal family $\mathcal{X} \rightarrow T_{g}$. We claim that $y$ has arbitrarily small neighborhoods $V$ with the following properties:

a) $V$ is $G_{y}$-stable;

b) for $y$ and $z$ in $V$, the fiber $\mathcal{X}_{y}$ is isomorphic to the fiber $\mathcal{X}_{z}$ if and only if $z=g y$, for some $g \in G_{y}$;

c) the stabilizer of $z$ is contained in $G_{y}$ for any $z \in V$.

In fact, we can take as $V$ the images $\alpha(B)$ of standard patches $\alpha: B \rightarrow T_{g}$ centered at $y$, corresponding to Kuranishi families $\mathcal{C} \rightarrow B$. The only one of the above properties that may not be clear is perhaps c). To prove it, recall that the action of $G_{y}$ on $V$ corresponds to the action on the base $B$ and total space $\mathcal{C}$ of the Kuranishi family of the automorphism group of the central fiber, and that every isomorphism between fibers of $\mathcal{C} \rightarrow B$ is induced by an automorphism of the central fiber. In particular, this is true of the automorphisms of the fiber above a point $b \in B$. Since the group of these automorphisms corresponds to the stabilizer of $z=\alpha(b)$, property c) follows.

We claim that a neighborhood satisfying properties a)-c) above also satisfies 2). Suppose in fact that $z=\gamma \cdot z^{\prime}$, where $z, z^{\prime} \in V$. It follows from b) that there is $\rho \in G_{y}$ such that $z^{\prime}=\rho \cdot z$. But then $\gamma \rho \in G_{z} \subset G_{y}$, and hence $\gamma$ belongs to $G_{y}$, as desired. We may now show that $T_{g}$ is Hausdorff. We already know that points $y$ and $y^{\prime}$ of $T_{g}$ belonging to different orbits of $\Gamma_{g}$ can be separated, and property 2) shows how to separate points belonging to the same orbit.

This completes the proof of the fact that $T_{g}$ is Hausdorff and that the mapping class group $\Gamma_{g}$ acts properly discontinuously on $T_{g}$.

We end this section by briefly discussing the definition of Teichmüller space. It is known that, if $X$ and $Y$ are surfaces without boundary, compact or not, two homeomorphisms $f_{1}, f_{2}: X \rightarrow Y$ are homotopic if and only if they are isotopic. A proof can be found in [18, cf. in particular Theorems 6.4 and A4]. An immediate consequence is that a Teichmüller structure on an unpointed curve $C$ can also be defined as the homotopy class of an orientation-preserving homeomorphism from $C$ to a reference surface $\Sigma$. Similarly, $\Gamma_{g}$ can be defined as the group of orientationpreserving homeomorphisms of $\Sigma$ modulo homotopy.

Another variant of the definitions of Teichmüller space and mapping class group, entirely equivalent to the original one, can be obtained by fixing a differentiable structure on the reference surface $\Sigma$ and replacing the words "homeomorphism" and "isotopy" with "diffeomorphism" and "differentiable isotopy" throughout. What this amounts to saying is that every class in $\Gamma_{g}$ contains a diffeomorphism, and that two diffeomorphisms which are isotopic are also differentiably isotopic. Thus the Teichmüller space $T_{g}$ is the set of isomorphism classes of objects $(C, \varphi)$, where $C$ is a curve of genus $g$ and $\varphi$ is the differentiable isotopy class of an oriented diffeomorphism from $C$ to $\Sigma$. Similarly,

$$
\Gamma_{g}=\operatorname{Diff}_{+}(\Sigma) / \operatorname{Diff}_{0}(\Sigma)
$$


where Diff ${ }_{+}(\Sigma)$ stands for the group of orientation-preserving diffeomorphisms of $\Sigma$ and $\operatorname{Diff}_{0}(\Sigma)$ for its identity component, which is nothing but the group of diffeomorphisms of $\Sigma$ which are differentiably isotopic to the identity.

\section{Continuous families of Riemann surfaces}

Roughly speaking, the universal property of the Kuranishi family of a smooth curve $C$ can be expressed by saying that any small deformation of $C$ can be obtained by pullback from the Kuranishi family. Our main goal in this section is to show that the same is true for "continuous deformations" of $C$.

First, we need some preliminaries. Let $\alpha: X \rightarrow S$ be a surjective morphism of $C^{m}$ manifolds, where $m=0, \ldots, \infty$. Suppose we can find an open cover $\left\{U_{i}\right\}$ of $X$ and $C^{m}$ diffeomorphisms $\Phi_{i}=\Phi_{i, 1} \times \Phi_{i, 2}: U_{i} \rightarrow V_{i} \times W_{i}$ of manifolds over $S$, where $V_{i}$ is open in $\mathbb{R}^{n}$ and $W_{i}$ is an open set in $S$, such that:

a) the function $v \mapsto \Phi_{i, 1} \circ \Phi_{j}^{-1}(v, w)$ is of class $C^{\infty}$ for any choice of $i$ and $j$ and for any $w \in W_{j}$,

b) the composition $\Phi_{i, 1} \circ \Phi_{j}^{-1}$ and all its derivatives, of any order, with respect to the the $V_{j}$ coordinates, are of class $C^{m}$, for any choice of $i$ and $j$.

We shall then say that the atlas $\mathcal{U}=\left\{\left(U_{i}, \Phi_{i}\right)\right\}$ defines on $\alpha: X \rightarrow S$ a structure of $C^{m}$ family of differentiable manifolds. We shall refer to the components of the functions $\Phi_{i, 1}$ as vertical coordinates and to derivatives with respect to them as vertical derivatives. We shall say that a function on an open set of $X$ is adapted if it is $C^{\infty}$ in the vertical coordinates and its vertical derivatives, of any order, are $C^{m}$; clearly, this notion does not depend on the chart in $\mathcal{U}$ with respect to which vertical derivatives are computed. Another atlas $\mathcal{U}^{\prime}$ will be considered equivalent to $\mathcal{U}$ if the adapted functions with respect to it are the same as the adapted functions with respect to $\mathcal{U}$. This is equivalent to asking that the vertical coordinates in either atlas be adapted with respect to the other atlas. We shall say that equivalent atlases define on $\alpha: X \rightarrow S$ the same structure of $C^{m}$ family of differentiable manifolds. Given a $C^{m}$ family of differentiable manifolds, the charts of any atlas defining the $C^{m}$ family structure will be said to be adapted. A morphism from a $C^{m}$ family of differentiable manifolds $\alpha: X \rightarrow S$ to another family $\beta: Y \rightarrow T$ is a commutative square

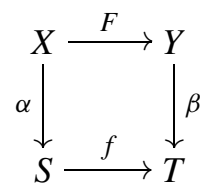

where $f$ and $F$ are $C^{m}$ morphisms and the composition of $F$ with any vertical coordinate on $Y$ is an adapted function. Clearly, the class of morphisms of $C^{m}$ families of differentiable manifolds is closed under composition. 
Let $\alpha: X \rightarrow S$ be a $C^{m}$ family of differentiable manifolds. There is an obvious notion of $C^{m}$ family of differentiable vector bundles (real or complex) on $\alpha: X \rightarrow S$. Examples are provided, for instance, by the relative tangent bundle to $X \rightarrow S$ and its dual. The class of $C^{m}$ families of differentiable vector bundles is closed under the standard vector bundle operations, such as passing to the dual, direct sum, tensor product, and exterior power. If $E$ is a $C^{m}$ family of differentiable vector bundles, it makes sense to speak of adapted local trivializations and adapted sections of $E$. In particular, it makes sense to speak of adapted relative differential forms (or, more generally, relative $E$-valued differential forms) along the fibers of $\alpha$. There is also a good notion of $C^{m}$ family of linear differential operators or, as we shall sometimes say, of adapted linear differential operator. Given two $C^{m}$ families $E$ and $E^{\prime}$ of differentiable vector bundles on $\alpha: X \rightarrow S$, a linear differential operator carrying sections of $E$ to sections of $E^{\prime}$ will be said to be a $C^{m}$ family of linear differential operators on $\alpha: X \rightarrow S$ if, when written in adapted coordinates and relative to adapted local trivializations of $E$ and $E^{\prime}$, it involves only differentiation with respect to vertical coordinates and its coefficients are adapted functions. Thus an adapted linear differential operator carries adapted sections to adapted sections.

We will need a basic result, due to Kodaira and Spencer [24], concerning the differentiability properties of solutions of differential equations $L u=v$, where $L$ is a $C^{m}$ family of differential operators and $v$ is an adapted section. Let $\alpha: X \rightarrow S$ be a $C^{m}$ family of compact differentiable manifolds, and let $E$ be a $C^{m}$ family of differentiable vector bundles on it. We denote by $\widetilde{A}(E)$ the vector space of adapted sections of $E$. Let $L: \widetilde{A}(E) \rightarrow \widetilde{A}(E)$ be a $C^{m}$ family of linear differential operators. A metric on $E$ will be said to be adapted if the inner product of any pair of adapted sections is an adapted functions. Adapted metrics always exist, and can for instance be constructed by gluing together flat local metrics by means of a partition of unity made up of adapted functions. Suppose an adapted metric is given on $E$, and one on the relative tangent bundle to $X \rightarrow S$. We denote by $\langle,\rangle_{s}$ the inner product on $E_{s}$, and by $d V_{s}$ the volume form on $X_{s}$ coming from the metric. Consider the inner product

$$
(u, v)=\int_{X_{s}}\langle u, v\rangle_{s} d V_{s}
$$

on $A\left(E_{S}\right)$, the vector space of $C^{\infty}$ sections of $E_{s}$. We will say that $L$ is a family of formally self-adjoint, strongly elliptic differential operators if each $L_{s}$ is selfadjoint with respect to the inner product (5.1), and strongly elliptic. Under these circumstances the kernel of $L_{S}$ is finite-dimensional, and there are linear operators

$$
F_{S}, G_{s}: A\left(E_{s}\right) \rightarrow A\left(E_{s}\right),
$$

where $F_{s}$ is the orthogonal projection onto the kernel of $L_{s}$, and

$$
u=F_{s} u+L_{S} G_{S} u .
$$

for any $u \in A\left(E_{s}\right)$. We shall refer to $F_{s}$ and $G_{s}$, respectively, as the harmonic projector and Green operator associated to $L_{s}$ and to the chosen metrics. The theorem of Kodaira and Spencer reads as follows. 
Theorem 5.1 ([24], Theorem 5). Let $m$ be a non-negative integer or $\infty$. Let $\alpha: X \rightarrow S$ be a $C^{m}$ family of compact differentiable manifolds, and let $E$ be a $C^{m}$ family of differentiable vector bundles on $\alpha: X \rightarrow S$. Suppose $E$ and the relative tangent bundle to $X \rightarrow S$ are endowed with adapted metrics. Let $L: \widetilde{A}(E) \rightarrow \widetilde{A}(E)$ be a $C^{m}$ family of formally self-adjoint, strongly elliptic linear differential operators. Suppose the dimension of the kernel of $L_{s}$ is independent of $s$. Then the family of harmonic projectors $F=\left\{F_{s}\right\}_{s \in S}$ and the family of Green operators $G=\left\{G_{s}\right\}_{s \in S}$ are of class $C^{m}$, in the sense that $F u$ and $G u$ are adapted, for any adapted $u$.

To be precise, the statement proved by Kodaira and Spencer is slightly less general than the one we have given, in two respects. First of all, they deal only with families of the form $X_{0} \times S \rightarrow S$, where $X_{0}$ is a compact manifold. More importantly, they treat only the case $m=\infty$. These, however, are not serious difficulties. Since the statement of Theorem 5.1 is local on $S$, the first is resolved by the following result.

Lemma 5.2. Let $\alpha: X \rightarrow S$ be a $C^{m}$ family of compact differentiable manifolds, let $E$ be a $C^{m}$ family of differentiable vector bundles on it, and let $s_{0}$ be a point of $S$. Then, if $U$ is a sufficiently small neighborhood of $s_{0}$, there is an isomorphism of $C^{m}$ families of differentiable manifolds between $\alpha^{-1}(U) \rightarrow U$ and the product family $X_{s_{0}} \times U \rightarrow U$. Moreover, if $q$ is the projection of $\alpha^{-1}(U) \cong X_{s_{0}} \times U$ to $X_{s_{0}}, E$ is isomorphic, as a $C^{m}$ family of differentiable vector bundles, to $q^{*}\left(E_{s_{0}}\right)$.

This result is well known, at least for $m=\infty$. However, since the usual proof, which involves integrating lifts to $X$ of coordinate vector fields on $S$, breaks down for $m=0$, we shall sketch an alternate proof below. Before we do so, however, we notice that the second difficulty mentioned above is also non-existent, since the proof given by Kodaira and Spencer for their theorem, and in particular their crucial Proposition 1, work equally well, and virtually without changes, in our context, provided we substitute Lemma 5.2 above, and in particular its second part, for their Lemma 1.

We now prove our Lemma 5.2. We shall use the following version "with parameters" of the inverse function theorem.

Lemma 5.3. Let $U$ be an open subset of $\mathbb{R}^{n}, V$ an open subset of $\mathbb{R}^{\ell}, f: U \times$ $V \rightarrow \mathbb{R}^{n}$ a continuous function, and $m$ a non-negative integer or $\infty$. Write $x=\left(x_{1}, \ldots, x_{n}\right)$ to indicate the standard coordinates in $U$ and $t=\left(t_{1}, \ldots, t_{\ell}\right)$ to indicate the standard ones in V. Suppose that:

i) the function $f$ is $C^{\infty}$ in $x$ for any fixed $t$;

ii) the function $f$ and all its derivatives, of any order, with respect to the $x$ variables, are $C^{m}$ functions of $x$ and $t$;

iii) the Jacobian $\frac{\partial f}{\partial x}(x, t)$ is non-singular at a point $\left(x_{0}, t_{0}\right) \in U \times V$.

Set $F(x, t)=(f(x, t), t)$. Then there is an open neighborhood $A$ of $\left(x_{0}, t_{0}\right)$ such that $F(A)$ is open in $\mathbb{R}^{n} \times \mathbb{R}^{\ell}$ and $F$ induces a homeomorphism from $A$ to $F(A)$. 
Moreover, writing the inverse of this function under the form $(x, t) \mapsto(g(x, t), t)$, the function $g$ is $C^{\infty}$ in $x$, and $g$ and all its derivatives, of any order, with respect to the $x$ variables, are $C^{m}$ functions of $x$ and $t$.

The proof of this lemma is essentially the same as the one of the standard inverse function theorem, and will not be given here.

Proof of Lemma 5.2. The first step is to show that families of compact differentiable manifolds can be locally embedded in euclidean space. More precisely, we shall see that, if $U$ is a sufficiently small neighborhood of $s_{0}$, for sufficiently large $N$ there is a map $F: \alpha^{-1}(U) \rightarrow \mathbb{R}^{N}$ such that the pair $\left(\mathbf{1}_{U}, F \times \alpha\right)$ is a morphism of $C^{m}$ families of differentiable manifolds from $\alpha^{-1}(U) \rightarrow U$ to $\mathbb{R}^{N} \times U \rightarrow U$ which is a fiberwise embedding. Denote by $B_{r}$ the ball of radius $r$ centered at the origin of $\mathbb{R}^{n}$. Shrinking $S$, if necessary, we may find finitely many adapted charts $\left(\varphi_{i}, \alpha\right): U_{i} \rightarrow B_{2} \times S$, where $U_{i}$ is an open subset of $X$, such that $X$ is covered by the open sets $\left(\varphi_{i}, \alpha\right)^{-1}\left(B_{1} \times S\right)$. Choose a non-negative $C^{\infty}$ function $\rho$ on $B_{2}$ which vanishes identically on the complement of $B_{4 / 3}$ and is identically equal to 1 on $B_{1}$. Pulling this back via $\varphi_{i}$ we get a function on $U_{i}$, which we denote by $\rho_{i}$. Write $\varphi_{i}=\left(\varphi_{i, 1}, \ldots, \varphi_{i, n}\right)$, and denote by $\psi_{i, j}$ the function $\rho_{i} \varphi_{i, j}$, extended to zero on the complement of $U_{i}$ in $X$; clearly, $\psi_{i, j}$ is adapted. Denote by $\psi$ the map from $X$ to $\mathbb{R}^{M}$ whose components are the $\psi_{i, j}$. The map $\psi$ is adapted, and its restriction to each fiber of $\alpha$ is a local embedding. In particular, there is an open neighborhood $W$ of the diagonal $\Delta$ in $X \times_{S} X$ such that $\psi(x) \neq \psi(y)$ for all $(x, y) \in W \backslash \Delta$. Shrinking $S$ again, we may find finitely many adapted charts $\left(\xi_{i}, \alpha\right): V_{i} \rightarrow B_{2} \times S$, where $V_{i}$ is an open subset of $X$, having the following property. We may select a set $I$ of pairs of indices such that $V_{i} \times{ }_{S} V_{j}$ does not meet $\Delta$ if $(i, j) \in I$, and $X \times{ }_{S} X \backslash W$ is covered by the open sets $\left(\xi_{i}, \alpha\right)^{-1}\left(B_{1} \times S\right) \times{ }_{S}\left(\xi_{j}, \alpha\right)^{-1}\left(B_{1} \times S\right)$ as $(i, j)$ varies in $I$. Denote by $\lambda_{i}$ the pullback of $\rho$ via $\xi_{i}$, extended to zero on the complement of $V_{i}$. By construction, if $(x, y) \in X \times{ }_{S} X \backslash W$, there is an index $i$ such that $\lambda_{i}(x) \neq 0$ but $\lambda_{i}(y)=0$. As a consequence, the $\lambda_{i}$, together with the $\psi_{i, j}$, are the components of an adapted map $X \rightarrow \mathbb{R}^{N}$ which is a fiberwise embedding.

We may thus view $X$ as embedded in $\mathbb{R}^{N} \times S$, and hence each fiber $X_{s}$ as embedded in $\mathbb{R}^{N}$. There is a neighborhood $V$ of $X_{s_{0}}$ in $\mathbb{R}^{N}$ which is diffeomorphic to a neighborhood of the zero section of the normal bundle to $X_{s_{0}}$ in $\mathbb{R}^{N}$, and the projection to the zero section in the normal bundle yields a $C^{\infty}$ map $\eta: V \rightarrow X_{s_{0}}$. Clearly, $X_{s} \subset V$ when $s$ is close to $s_{0}$, and therefore, after suitably shrinking $S, \eta$ gives an adapted map $\beta: X \rightarrow X_{S_{0}}$. We wish to show that, possibly after further shrinking $S,(\beta, \alpha): X \rightarrow X_{s_{0}} \times S$ is an isomorphism of $C^{m}$ families of differentiable manifolds. As the embedding of $X$ in $\mathbb{R}^{N}$ is adapted, the tangent spaces to the $X_{s}$ vary continuously. Moreover, Lemma 5.3 implies that every point of $X_{s_{0}}$ has a neighborhood $U$ such that $\beta$ induces a diffeomorphism from $X_{s} \cap \beta^{-1}(U)$ to $U$ for $s$ near $s_{0}$. Let us see that, in fact, $\beta_{\mid X_{s}}$ is a diffeomorphism when $s$ is close to $s_{0}$. It suffices to prove injectivity. Suppose there are a sequence $\left\{s_{n}\right\}$ in $S$ converging to $s_{0}$ and sequences of points $x_{n}, y_{n} \in X_{s_{n}}$ such that $x_{n} \neq y_{n}$ but $\beta\left(x_{n}\right)=\beta\left(y_{n}\right)$; we may assume that $\left\{x_{n}\right\}$ and $\left\{y_{n}\right\}$ converge, respectively, to 
points $x, y \in X_{s_{0}}$. Letting $n$ go to infinity we find that $x=\beta(x)=\beta(y)=y$. This shows that, if $U$ is neighborhood of $x$ as above, $x_{n}$ and $y_{n}$ belong to $U$ for large $n$, and hence $\beta\left(x_{n}\right) \neq \beta\left(y_{n}\right)$, a contradiction. This proves the first part of Lemma 5.2.

The proof of the last statement is similar. We sketch it for $E$ a real vector bundle, the proof for a complex bundle being entirely analogous. First notice that, up to shrinking $S$, there exist enough adapted sections of $E$ to embed $E$ as a subvector bundle in a trivial bundle $\mathbb{R}^{K} \times X_{s_{0}} \times S$, for some large $K$. The orthogonal projection with respect to the Euclidean metric of $\mathbb{R}^{K}$ gives a surjective morphism of $C^{\infty}$ vector bundles on $X_{s_{0}}$ from $\mathbb{R}^{K} \times X_{s_{0}}$ to $E_{s_{0}}$. As a consequence, we get a surjective morphism $\mathbb{R}^{K} \times X_{s_{0}} \times S \rightarrow q^{*}\left(E_{S_{0}}\right)$ which, when composed with the inclusion of $E \subset \mathbb{R}^{K} \times X_{s_{0}} \times S$ yields a morphism of adapted vector bundles $E \rightarrow q^{*}\left(E_{S_{0}}\right)$. The restriction of this to $\alpha^{-1}(U)$ is an isomorphism for any sufficiently small neighborhood $U$ of $s_{0}$.

The notion of $C^{m}$ family of differentiable manifolds has a holomorphic counterpart in the one of $C^{m}$ family of complex manifolds. Formally, such a family is a surjective morphism $\alpha: X \rightarrow S$ of $C^{m}$ manifolds with the property that each fiber $X_{S}$ is a complex manifold, satisfying the following local triviality condition. For every $x \in X$ there is a $C^{m}$ diffeomorphism $\varphi: U \rightarrow V \times W$, where $U$ is a neighborhood of $x$ in $X, W$ is a neighborhood of $\alpha(x)$ in $S$, and $V$ is a ball centered at 0 in some $\mathbb{C}^{h}$, such that:

i) $\varphi$ is compatible with the projections to $S$;

ii) $\varphi(x)=(0, \alpha(x))$;

iii) $\varphi$ maps $U \cap \alpha^{-1}(s)$ biholomorphically onto $V \times\{s\}$ for every $s \in W$.

When all the fibers of $\alpha$ are curves we will speak of $C^{m}$ family of curves. A $C^{m}$ family of compact complex manifolds has a natural structure of $C^{m}$ family of differentiable manifolds, as a consequence of the following standard result.

Lemma 5.4. Let $f\left(z_{1}, \ldots, z_{h}, t_{1}, \ldots, t_{\ell}\right)$ be a $C^{m}$ function of the complex variables $z_{1}, \ldots, z_{h}$ and of the real variables $t_{1}, \ldots, t_{\ell}$ which is holomorphic in $z_{1}, \ldots, z_{h}$. Then the partials of $f$, of any order, with respect to the variables $z_{1}, \ldots, z_{h}$, are $C^{m}$ functions of $z_{1}, \ldots, z_{h}, t_{1}, \ldots, t_{\ell}$.

The lemma follows directly from Cauchy's integral formula

$$
\begin{aligned}
& \frac{\partial^{\sum k_{i}}}{\partial z_{1}^{k_{1}} \ldots \partial z_{h}^{k_{h}}} f\left(z_{1}, \ldots, z_{h}, t_{1}, \ldots, t_{k}\right) \\
& \quad=\frac{\prod k_{i} !}{(2 \pi i)^{h}} \int_{\substack{\left|\zeta_{i}-z_{i}\right|=\varepsilon \\
i=1, \ldots, h}} \frac{f\left(\zeta_{1}, \ldots, \zeta_{h}, t_{1}, \ldots, t_{\ell}\right)}{\prod\left(\zeta_{i}-z_{i}\right)^{k_{i}+1}} d \zeta_{1} \ldots d \zeta_{h} .
\end{aligned}
$$

In fact, the right-hand side can be continuously differentiated $m$ times under the integral sign with respect to the variables $t_{i}$. 
A morphism of $C^{m}$ families of complex manifolds is just a morphism of $C^{m}$ families of differentiable manifolds which happens to be holomorphic on the fibers. If $\alpha: X \rightarrow S$ is a $C^{m}$ family of complex manifolds, we denote by $\widetilde{\mathcal{O}}_{X}$ the sheaf of $C^{m}$ functions on $X$ which are holomorphic along the fibers, and by $\widetilde{\Omega}_{\alpha}^{p}$ the $\widetilde{\mathcal{O}}_{X}$-module whose sections are the relative differential forms which restrict to a holomorphic $(p, 0)$-form on each fiber. We also write $\widetilde{\mathcal{A}}_{\alpha}^{p, q}$ for the sheaf of adapted relative $(p, q)$-forms; it is a module over $\widetilde{\mathcal{A}}_{X}$, the sheaf of adapted functions. Now let $C$ be a curve. A $C^{m}$ deformation of $C$ is the datum of a pointed $C^{m}$ manifold $\left(S, s_{0}\right)$, a $C^{m}$ family of curves $\alpha: X \rightarrow S$, and an isomorphism $\varphi: C \stackrel{\simeq}{\rightarrow} X_{s_{0}}$. A morphism of $C^{m}$ deformations from $\alpha: X \rightarrow\left(S, s_{0}\right), \varphi: C \rightarrow X_{s_{0}}$ to another deformation $\alpha^{\prime}: X^{\prime} \rightarrow\left(S^{\prime}, s_{0}^{\prime}\right), \varphi^{\prime}: C \rightarrow X_{s_{0}^{\prime}}^{\prime}$ is a pair $(f, F)$ of $C^{m}$ morphisms fitting in a Cartesian diagram

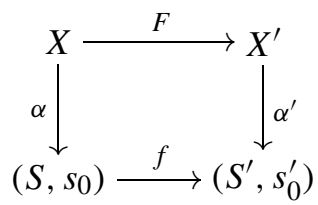

such that $(f, F)$ is a morphism of $C^{m}$ families of curves and $F_{S_{0}} \circ \varphi=\varphi^{\prime}$. We shall show that the universal property of the Kuranishi family of $C$ holds also with respect to $C^{m}$ deformations.

Proposition 5.5. Let $C$ be a curve of genus $g>1$, and let $\alpha: X \rightarrow\left(S, s_{0}\right)$, $\varphi: C \rightarrow X_{s_{0}}$, be a $C^{m}$ deformation of $C$. Let $\pi: \mathcal{C} \rightarrow\left(B, b_{0}\right), \psi: C \rightarrow \mathcal{C}_{b_{0}}$, be a Kuranishi family for $C$. Then, for some open neighborhood $A$ of $s_{0}$, there is a morphism $(f, F)$ of $C^{m}$ deformations from $\alpha^{-1}(A) \rightarrow\left(A, s_{0}\right), \varphi: C \rightarrow X_{s_{0}}$ to the Kuranishi family. This morphism is essentially unique, in the sense that any morphism of deformations from $\alpha^{-1}\left(A^{\prime}\right) \rightarrow\left(A^{\prime}, s_{0}\right), \varphi: C \rightarrow X_{s_{0}}, A^{\prime}$ a neighborhood of $s_{0}$, to the Kuranishi family, agrees with $(f, F)$ on $\alpha^{-1}(U) \rightarrow U$, where $U$ is a neighborhood of $s_{0}$.

Proof. We claim that, possibly after shrinking $S$, we can find a Cartesian diagram

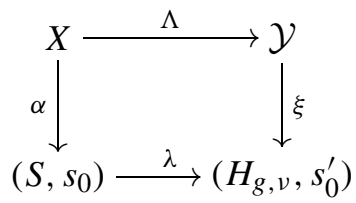

where $(\lambda, \Lambda)$ is a morphism of $C^{m}$ families of complex manifolds and $\xi: \mathcal{Y} \rightarrow$ $H_{g, v}$ is the universal family over the Hilbert scheme of $v$-canonically embedded genus $g$ curves, for some $v \geq 3$. The existence part of the proposition then follows from the standard universal property of the Kuranishi family, applied to $\mathcal{Y} \rightarrow H_{g, v}$.

To prove the existence of (5.3) we proceed as follows. Let $u_{s_{0}}$ be a holomorphic $v$-fold differential on $X_{s_{0}}$, for some $v \geq 3$. We shall show that $u_{s_{0}}$ extends, 
possibly after shrinking $S$, to a section of $\mathcal{L}=\left(\widetilde{\Omega}_{\alpha}^{1}\right)^{\otimes v}$ on all of $X$. First of all, by Lemma $5.2, u_{s_{0}}$ extends to a section $u$ of $\left(\widetilde{\mathcal{A}}_{\alpha}^{1,0}\right)^{\otimes v}=\mathcal{L} \otimes_{\widetilde{\mathcal{O}}_{X}} \widetilde{\mathcal{A}}_{X}$. Put adapted metrics on the $C^{m}$ families of differentiable vector bundles underlying $\mathcal{L}$ and $\widetilde{\mathcal{A}}_{\alpha}^{1,0}$. For each $s \in S$, we write $u_{s}$ for the restriction of $u$ to the fiber $X_{s}$, and $\bar{\partial}_{s}$ to indicate the $\bar{\partial}$ operator acting on sections of $\mathcal{L} \otimes_{\widetilde{\mathcal{O}}_{X}} \mathcal{A}_{X_{s}}$. We also let $\vartheta_{s}$ be the formal adjoint of $\bar{\partial}_{s}$, and $\square_{s}=\bar{\partial}_{s} \vartheta_{s}$ the Laplace-Beltrami operator, both acting on $\mathcal{L}$-valued $(0,1)$ forms on $X_{s}$. The kernel of $\square_{s}$ can be identified with $H^{1}\left(X_{s}, \mathcal{O}\left(K_{X_{s}}^{v}\right)\right.$ ), where $K_{X_{s}}$ stands for the canonical bundle of $X_{s}$, and hence is reduced to zero, since $g>1$ and $v \geq 3$. In particular, the family of differential operators $\left\{\square_{s}\right\}$ satisfies the assumptions of Theorem 5.1. It follows from (5.2) that

$$
\bar{\partial}_{s} u_{s}=\square_{s} G_{s} \bar{\partial}_{s} u_{s}=\bar{\partial}_{s} \vartheta_{s} G_{s} \bar{\partial}_{s} u_{s}
$$

By Theorem 5.1, $\left\{G_{s} \bar{\partial}_{s} u_{s}\right\}$, and hence $v=\left\{v_{s}\right\}=\left\{\vartheta_{s} G_{s} \bar{\partial}_{s} u_{s}\right\}$, are adapted. Since $\bar{\partial}_{s}\left(u_{s}-v_{s}\right)=0$ for any $s$, and $v_{s_{0}}=0$, we conclude that $u-v$ is a section of $\mathcal{L}$ extending $u_{s_{0}}$.

Now choose a basis for the space of holomorphic $v$-fold differentials on $X_{s_{0}}$ and, after suitably shrinking $S$, extend its elements to sections $u_{0}, \ldots, u_{N}$ of $\mathcal{L}$ on $X$, where $N=(2 v-1)(g-1)-1$. We may assume that the restrictions of these sections to $X_{S}$ constitute a basis for the space of holomorphic $\nu$-fold differentials on $X_{s}$, for any $s$. The sections $u_{0}, \ldots, u_{N}$ then give a morphism of $C^{m}$ families of complex manifolds over $S$

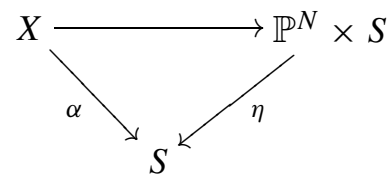

such that $X_{s} \rightarrow \mathbb{P}^{N}$ is a $v$-canonical embedding for each $s$. To construct (5.3), we take as $\lambda$ the map which associates to each $s \in S$ the point of $H_{g, v}$ corresponding to $X_{s} \hookrightarrow \mathbb{P}^{N}$. Recall that $\lambda(s)$ can be described as follows. The pullback homomorphism

$$
H^{0}\left(\mathbb{P}^{N}, \mathcal{O}(k)\right) \rightarrow H^{0}\left(X_{s}, \mathcal{O}_{X_{s}}(k)\right) \cong H^{0}\left(X_{s}, \mathcal{O}\left(K_{X_{s}}^{k v}\right)\right)
$$

is onto for every $k \geq 1$, by Noether's theorem. Its kernel is a point of the Grassmannian $\mathbb{G}$ of $\left(\left(\begin{array}{c}N+k \\ N\end{array}\right)-(2 k v-1)(g-1)\right)$-planes in $\left(\begin{array}{c}N+k \\ N\end{array}\right)$-space. For large enough $k$, the Hilbert scheme $H_{g, v}$ is a subscheme of $\mathbb{G}$, and $\lambda(s)$ is just the kernel of (5.4). On the other hand, by construction, the homomorphism (5.4) is the fiber at $s$ of a homomorphism of $C^{m}$ vector bundles

$$
\eta_{*} \widetilde{\mathcal{O}}_{\mathbb{P}^{N} \times S}(k) \rightarrow \alpha_{*} \mathcal{L}^{k}
$$

Since the kernel of this homomorphism is a $C^{m}$ vector subbundle of the trivial bundle on the left, the map $\lambda$ is $C^{m}$. 
The map $\Lambda$ is easily constructed. If $x$ is a point of $X$, then

$$
\Lambda(x)=\left(\left[u_{0}(x): \cdots: u_{N}(x)\right], \lambda(s)\right) \in X^{\prime} \subset \mathbb{P}^{N} \times H_{g, v} .
$$

It is clear that $(\lambda, \Lambda)$ is a morphism of $C^{m}$ families.

It remains to prove the uniqueness property of $(f, F)$. Let $\left(f^{\prime}, F^{\prime}\right)$ be another morphism of deformations from $\alpha^{-1}\left(A^{\prime}\right) \rightarrow\left(A^{\prime}, s_{0}\right), \varphi: C \rightarrow X_{s_{0}}$ to the Kuranishi family of $C$. We may suppose that $A^{\prime}=A$. We may also suppose that $\operatorname{Aut}(C)$ acts on $\mathcal{C}$ and $B$, and that any isomorphism between fibers of $\pi$ comes from an automorphism of $C$. Assume that there is a sequence $\left\{x_{n}\right\}$ in $A$, converging to $s_{0}$, such that the restriction of $F$ to $X_{x_{n}}$ is different from the one of $F^{\prime}$ for each $n$. We will show that this leads to a contradiction. In fact, what the assumption implies is that for each $n$ there is a non-trivial element $\gamma_{n}$ of $\operatorname{Aut}(C)$ such that $\gamma_{n} f\left(x_{n}\right)=f^{\prime}\left(x_{n}\right)$ and $\gamma_{n} F$ coincides with $F^{\prime}$ on $X_{x_{n}}$. As the automorphism group of $C$ is finite, we may suppose that all the $\gamma_{n}$ are equal to a fixed $\gamma \in \operatorname{Aut}(C)$. Then, passing to the limit for $n \rightarrow \infty$, we conclude that $\gamma F$ and $F^{\prime}$ agree also on $X_{s_{0}}$. On the other hand, since we are dealing with morphisms of deformations, $F_{s_{0}} \circ \varphi=F_{s_{0}}^{\prime} \circ \varphi$, which implies that $\gamma=1$, a contradiction.

The notion of Teichmüller structure carries over, with obvious changes, to the context of $C^{m}$ families of curves. An important corollary of Proposition 5.5 is then the following.

Theorem 5.6. Let $\alpha: X \rightarrow S$ be a $C^{m}$ family of genus $g$ curves with Teichmüller structure. Suppose $g \geq 2$. Let $\eta: \mathcal{X}_{g} \rightarrow T_{g}$ be the universal family on the genus $g$ Teichmüller space. Let $f: S \rightarrow T_{g}$ be the map which associates to each point of $S$ the isomorphism class of the corresponding fiber, and let $F: X \rightarrow \mathcal{X}_{g}$ be the map whose restriction to the fiber $X_{S}$ is the unique isomorphism of curves with Teichmüller structure from $X_{s}$ to $\eta^{-1}(f(s))$. Then the pair $(f, F)$ is a morphism of $C^{m}$ families of curves. In particular, $f$ is of class $C^{m}$.

\section{The theorem of Teichmüller}

In this section we discuss the following famous theorem of Teichmüller.

Theorem 6.1. Let $g>1$. The Teichmüller space $T_{g}$ is homeomorphic to the unit ball in $\mathbb{C}^{3 g-3}$.

Our plan is to show how the proof of this theorem directly reduces to the proof of two fundamental results, namely Teichmüller's uniqueness theorem and the theorem of existence of solutions of the Beltrami equation.

Before we can proceed, we must introduce the notion of Beltrami differential. Let $S$ be a compact connected Riemann surface. We consider vector-valued differentials on $S$ which are locally of the form

$$
\mu=v \frac{\partial}{\partial z} \otimes d \bar{z}
$$


where $z$ is a local coordinate and $v$ is a measurable function, that is, measurable sections of $T_{S} \otimes \bar{K}_{S}$, where $T_{S}$ and $K_{S}$ are, respectively, the complex tangent and cotangent bundles to $S$. It makes sense to define a measurable function $|\mu|$ by setting it locally equal to $|\nu|$, since the latter is clearly independent of the choice of coordinate $z$. A Beltrami differential on the Riemann surface $S$ is an $L^{\infty}$ section of $T_{S} \otimes \bar{K}_{S}$ whose norm $\|\mu\|=\sup _{S}|\mu|$ is strictly less than 1 . We shall really need only a particular kind of Beltrami differentials, namely those which are $C^{\infty}$ everywhere, except at a finite number of points. These differentials will be called admissible.

To a Beltrami differential we associate a perturbed version of the $\bar{\partial}$ operator on $S$, by setting

$$
\bar{\partial}_{\mu}=\bar{\partial}-\mu,
$$

where $\mu$ acts on a function $f$ as

$$
\mu(f)=v \frac{\partial f}{\partial z} d \bar{z} .
$$

The corresponding Beltrami equation is

$$
\bar{\partial}_{\mu} u=0,
$$

that is,

$$
u_{\bar{z}}=v(z) u_{z} .
$$

The basic existence theorem for the Beltrami equation asserts that it has local solutions, in an appropriate generalized sense, which are homeomorphisms to open subsets of the complex plane. We will not need the full strength of this result, which is due to Morrey [29], but just the fact that the same conclusion holds under the stronger hypothesis that $\mu$ is $C^{\infty}$. This is due to Korn [25] and Lichtenstein [27], who more generally deal with the case when $\mu$ satisfies a Hölder condition; a simplified proof of their result was given by Bers [7] and Chern [14], and can be found also in [15, Chapter IV, Section 8]. Formally, the existence result we need is the following.

Theorem 6.2. Let $v\left(z, t_{1}, \ldots, t_{n}\right)$ be a $C^{\infty}$ function on a neighborhood of the origin in $\mathbb{C} \times \mathbb{R}^{n}$. Suppose that $|v(z, t)|<1$ for all values of $z$ and $t=\left(t_{1}, \ldots, t_{n}\right)$. Then there exists a $C^{\infty}$ function $w(z, t)$, also defined on a neighborhood of the origin, such that

$$
w_{\bar{z}}=v(z, t) w_{z}, \quad w_{z}(0,0) \neq 0 .
$$

We shall also need the following uniqueness result.

Lemma 6.3. Let $v(z)$ be a function on a neighborhood of the origin in the complex plane which is $C^{\infty}$ except at a finite set Z. Suppose that $|v(z)|<1$ for all $z$. Let $u$ be a homeomorphism from a neighborhood of the origin to an open subset of $\mathbb{C}$ which solves the Beltrami equation (6.1) away from $Z$. Let $f$ be a bounded function on a neighborhood of the origin which is once differentiable away from $Z$. Then $f$ satisfies (6.1) away from $Z$ if and only if it is a holomorphic function of $u$. 
The proof of the lemma is quite straightforward. Suppose first that $Z$ is empty, and let $w$ be the solution of the Beltrami equation provided by Theorem 6.2. A simple chain rule computation shows that

$$
f_{\bar{z}}-v f_{z}=\left(1-|v|^{2}\right) \cdot f_{\bar{w}} \cdot \overline{w_{z}} .
$$

Thus $f$ is a holomorphic function of $w$ near the origin if and only if it is a solution of the Beltrami equation. This applies in particular to the function $u$. Moreover, since $u$ has an inverse, it is also the case that $w$ is a holomorphic function of $u$. This proves the lemma when $Z$ is empty. In the general case what the above argument shows is that $f$ solves the Beltrami equation away from $Z$ if and only if it is a holomorphic function of $u$ there. Holomorphicity of $f$ at points of $Z$ then follows from the Riemann extension theorem.

Theorem 6.2 in its parameterless version, that is, for $n=0$, and Lemma 6.3 say, in particular, that a $C^{\infty}$ Beltrami differential $\mu$ on a Riemann surface $S$ defines on $S$ a new complex structure whose holomorphic functions are the solutions of the corresponding Beltrami equation. The reader should be warned that $\bar{\partial}_{\mu}$ is not the $\bar{\partial}$ operator of this complex structure, but just its component of type $(0,1)$ (with respect to the original structure). A more suggestive, though cumbersome, notation for $\bar{\partial}_{\mu}$ could thus be $\bar{\partial}_{\mu}^{(0,1)}$.

Dependence on parameters in Theorem 6.2 is often not considered in the literature; a notable exception is [2], to which we might refer for a proof. A cheap alternative is to appeal instead to Theorem 5.1. Here is how the argument goes. Since the problem is of a local nature, we may alter $v$ outside a neighborhood of the origin. Hence we may assume that $v$ is defined and $C^{\infty}$ on $\mathbb{C} \times U$, where $U$ is a neighborhood of the origin in $\mathbb{R}^{n}$, and that there is a positive $r$ such that $v$ vanishes for $|z|>r$. Thus we may view $\mu=v \partial / \partial z \otimes d \bar{z}$ as a family $\left\{\mu_{t}\right\}_{t \in U}$ of Beltrami differentials on $\mathbb{P}^{1}$, vanishing outside the disk of radius $r$ centrered at 0 . Let $H$ be the hyperplane bundle on $\mathbb{P}^{1}$; its smooth sections can be viewed as $C^{\infty}$ functions $f$ on $\mathbb{C}$ such that $f / z$ extends in a $C^{\infty}$ way across $\infty$. Since $\bar{\partial}_{\mu_{t}}$ is the standard $\bar{\partial}$ operator in a neighborhood of $\infty$, for any smooth section $u$ of $H, \bar{\partial}_{\mu_{t}} u$ is a smooth $H$-valued $(0,1)$-form. Let $\vartheta_{\mu_{t}}$ be the formal adjoint of $\bar{\partial}_{\mu_{t}}$ with respect to (say) the Fubini-Study metric. Then the differential operator $L_{t}=\vartheta_{\mu_{t}} \bar{\partial}_{\mu_{t}}$ is self-adjoint and strongly elliptic. If $u$ is a section of $H$ such that $L_{t} u=0$, then $\left(\bar{\partial}_{\mu_{t}} u, \bar{\partial}_{\mu_{t}} u\right)=\left(u, L_{t} u\right)=0$, and hence $\bar{\partial}_{\mu_{t}} u=0$. In other words, the $L_{t^{-}}$ harmonic sections of $H$ are just those sections which are holomorphic with respect to the complex structure defined by $\mu_{t}$. The space of these sections has dimension 2 for any $t$, by the Riemann-Roch theorem, so that Theorem 5.1 applies. Pick a $\mu_{0}$-holomorphic section $u$ which vanishes simply at 0 , and set $w_{t}=F_{t} u$, where $F_{t}$ is the harmonic projector associated to $L_{t}$. Then $\bar{\partial}_{\mu_{t}} w_{t}=0$, and $w_{t}$ depends differentiably on $t$, by Theorem 5.1. In other words, $w(z, t)=w_{t}(z)$ has all the required properties.

Admissible Beltrami differentials originate, in particular, from the so-called admissible quasi-diffeomorphisms. An orientation-preserving homeomorphism 
$F: S \rightarrow S^{\prime}$ between two compact Riemann surfaces which is a diffeomorphism outside a finite set $Z \subset S$ is called a quasi-diffeomorphism. Pick holomorphic coordinates $z$ and $w$ around $p \in S \backslash Z$ and $F(p) \in S^{\prime}$, respectively. The condition that $F$ be an orientation-preserving diffeomorphism on $S \backslash Z$ tells us that, on $S \backslash Z$, the Jacobian determinant of $F$ is positive. Since, in local coordinates, the Jacobian is

$$
\left|w_{z}\right|^{2}-\left|w_{\bar{z}}\right|^{2}
$$

the local function

$$
v(z)=\frac{w_{\bar{z}}}{w_{z}}
$$

is $C^{\infty}$ away from $Z$ and of absolute value less than 1. It is a straightforward application of the chain rule to check that setting

$$
\mu_{F}=v \frac{\partial}{\partial z} \otimes d \bar{z}
$$

gives a well-defined section of $T_{S} \otimes \bar{K}_{S}$ which is $C^{\infty}$ away from $Z$. The quasidiffeomorphism $F$ is said to be admissible if $\left\|\mu_{F}\right\|<1$, i.e., if $\mu_{F}$ is a Beltrami differential. By Lemma 6.3, the complex structure of $S^{\prime}$ can be completely described in terms of the one of $S$ and of the differential $\mu_{F}$; a bounded function $f$ on an open subset of $S^{\prime}$ is holomorphic if and only if $u=f \circ F$ is a solution of the Beltrami equation $\bar{\partial}_{\mu_{F}} u=0$ away from $Z$.

Lemma 6.4. Let $F: S \rightarrow S^{\prime}$ be a quasi-diffeomorphism. Then

$$
\left\|\mu_{F}\right\|=\left\|\mu_{F^{-1}}\right\| .
$$

If $F^{\prime}: S \rightarrow S^{\prime \prime}$ is another quasi-diffeomorphism, then $\mu_{F^{\prime}}=\mu_{F}$ if and only if $F^{\prime} \circ F^{-1}: S^{\prime} \rightarrow S^{\prime \prime}$ is holomorphic.

The second assertion of the lemma follows immediately from Lemma 6.3, while another elementary chain rule computation shows that $\left|\mu_{F}(p)\right|=\left|\mu_{F^{-1}}(F(p))\right|$ for any $p \in S$, thus proving the first assertion.

It is convenient to introduce the concept of dilatation for a quasi-diffeomorphism $F: S \rightarrow S^{\prime}$. This is simply defined to be

$$
K[F]=\frac{1+\left\|\mu_{F}\right\|}{1-\left\|\mu_{F}\right\|} .
$$

It follows from Lemma 6.4 that a quasi-diffeomorphism and its inverse have the same dilatation. It is also clear that $F$ is admissible if and only if $K[F]<\infty$.

We now turn to Theorem 6.1. Let $S$ be a reference Riemann surface of genus $g>1$. As we have announced, we shall rely on a fundamental result of Teichmüller, the so-called Teichmüller uniqueness theorem. This provides a canonical representative for each isotopy class of orientation-preserving homeomorphisms $f: C \rightarrow S$, which has the following two remarkable properties:

1. it is an admissible quasi-diffeomorphism; 
2. away from the points where it fails to be smooth it can be locally described, in a canonical way, as a real affine transformation.

Let $\omega$ be a holomorphic quadratic differential on $S$. If $\omega=f(z) d z^{2}$ is a local expression for it in terms of a local coordinate $z$, we define the (singular) volume form associated to $\omega$ to be

$$
d A_{\omega}=\frac{i}{2}|f| d z \wedge d \bar{z}=|f| d x \wedge d y,
$$

where $x$ and $y$ stand for the real and imaginary parts of $z$. One immediately checks that this definition is independent of the choice of local coordinate. Now consider the $(3 g-3)$-dimensional space $H^{0}\left(S, \mathcal{O}\left(K_{S}^{2}\right)\right)$ of holomorphic quadratic differentials on $S$. A norm is introduced in this space by setting

$$
\|\omega\|=\int_{S} d A_{\omega}
$$

for any $\omega$ in $H^{0}\left(S, \mathcal{O}\left(K_{S}^{2}\right)\right)$. Look at the unit ball in $H^{0}\left(S, \mathcal{O}\left(K_{S}^{2}\right)\right)$ :

$$
B\left(K_{S}^{2}\right)=\left\{\omega \in H^{0}\left(S, \mathcal{O}\left(K_{S}^{2}\right)\right):\|\omega\|<1\right\} .
$$

We are going to define a map

$$
\Phi: B\left(K_{S}^{2}\right) \rightarrow T_{S}=T_{g}
$$

and prove the following more precise version of (6.1).

Theorem 6.5. $\Phi$ is a homeomorphism.

The construction of $\Phi$ is as follows. Pick $\omega \in B\left(K_{S}^{2}\right)$ and set

$$
k=\|\omega\|<1 .
$$

Let $Z$ be the set of zeroes of $\omega$. We introduce a new complex structure on $S \backslash Z$. Near a point $p \in S \backslash Z$ we may write

$$
\omega=(d z)^{2},
$$

where $z$ is a holomorphic local coordinate, which is uniquely defined up to sign and the addition of a constant. We will say that $z$ is an $\omega$-coordinate. We define a new coordinate patch around $p$ by setting

$$
z^{\prime}=\frac{z+k \bar{z}}{1-k} .
$$

A different choice of $z$ changes $z^{\prime}$ by at most a sign and the addition of a constant. Hence these coordinate patches define a new holomorphic structure on $S \backslash Z$. We 
will show that this structure extends to one on all of $S$ by constructing explicit coordinate patches around each point of $Z$; this extension will clearly be unique. Let $p$ be a zero of $\omega$, and $z$ a local coordinate around $p$. Write $\omega=f(z) d z^{2}$ near $p$. We first treat the case when $f$ vanishes to even order $2 n$ at $p$. By suitably changing coordinate, we may suppose that $\omega=d\left(z^{n+1}\right)^{2}$. It follows that $z^{n+1}$ is an $\omega$-coordinate at all points of $S$ sufficiently close to $p$, but different from it, and hence, by virtue of (6.2), that

$$
\xi(z)=\frac{z^{n+1}+k \bar{z}^{n+1}}{1-k}
$$

is a local coordinate for the new complex structure on $S \backslash Z$ at all these points. Since $k<1$, the function $1+k \frac{\bar{z}^{n+1}}{z^{n+1}}$ takes its values in the half-plane of complex numbers with positive real part, where a single-valued determination of the $(n+1)$-st root function exists. Set

$$
\eta(z)=z\left(\frac{1+k \frac{\bar{z}^{n+1}}{z^{n+1}}}{1-k}\right)^{\frac{1}{n+1}}
$$

for $z \neq 0$ and $\eta(0)=0$. Since $1+k \frac{z^{n+1}}{z^{n+1}}$ is bounded, the function $\eta$ is continuous at $p$. We will now show that $\eta$ is a homeomorphism between an open neighborhood of $p$ and an open neighborhood of the origin in the complex plane, and we will take it as our new coordinate around $p$. This is compatible with the new complex structure on $S \backslash Z$, since $\eta^{n+1}=\xi$. To prove our claim, by the "invariance of domain" theorem, it suffices to show that $\eta$ is injective. If $\eta(z)=\eta\left(z^{\prime}\right)$, then $\xi(z)=\xi\left(z^{\prime}\right)$, and hence $z^{n+1}=z^{\prime n+1}$. It follows that

$$
1+k \frac{\bar{z}^{n+1}}{z^{n+1}}=1+k \frac{{\overline{z^{\prime}}}^{n+1}}{z^{\prime n+1}}
$$

and therefore that $z=z^{\prime}$, by the definition of $\eta$. We now treat the case when $f$ vanishes to odd order $m$ at $p$. Let $q$ be the map $\zeta \mapsto \zeta^{2}=z$. The pulled-back differential $q^{*} \omega$ vanishes to order $2 m+2$ at the origin, and by suitably changing coordinate we may suppose that $q^{*} \omega=d\left(\zeta^{m+2}\right)^{2}$. This means that $\zeta^{m+2}$ is an $\omega$-coordinate at all points of $S$ close to $p$, but different from it. Thus

$$
\xi=\frac{\zeta^{m+2}+k \bar{\zeta}^{m+2}}{1-k}
$$

is a local coordinate for the new complex structure on $S \backslash Z$ at these points. We take as new coordinate at $p$ the function $\eta(z)$ whose value is

$$
\eta(z)=z\left(\frac{1+k \frac{|z|^{m+2}}{z^{m+2}}}{1-k}\right)^{\frac{2}{m+2}}=\zeta^{2}\left(\frac{1+k \frac{\bar{\zeta}^{m+2}}{\zeta^{m+2}}}{1-k}\right)^{\frac{2}{m+2}}
$$


for $z \neq 0$ and zero for $z=0$. To prove that this extends the new complex structure on $S \backslash Z$ one proceeds as in the even order case, after noticing that $\eta^{m+2}=\xi^{2}$. This finishes the construction of the new complex structure of $S$.

We may now define the Teichmüller map $\Phi: B\left(K_{S}^{2}\right) \rightarrow T_{g}$. We set

$$
\Phi(\omega)=\left[S_{\omega},\left[f_{\omega}\right]\right],
$$

where $S_{\omega}$ is the surface $S$, equipped with the new complex structure we have just described, and $f_{\omega}: S_{\omega} \rightarrow S$ is the set-theoretic identity. The homeomorphism $f_{\omega}$ is called the Teichmüller map associated to $\omega$, and is an admissible quasidiffeomorphism. In fact, by (6.2), $v_{f_{\omega}}=-k$, and hence

$$
\left|\mu_{f_{\omega}}\right|=k=\|\omega\|<1
$$

everywhere.

It is crucial to observe that $S_{\omega}$ and $f_{\omega}$ depend continuously on $\omega \in B\left(K_{S}^{2}\right)$. More precisely, if we denote by $\mathcal{S}$ the disjoint union of all the $S_{\omega}$ and by $f$ the map from $\mathcal{S}$ to $S \times B\left(K_{S}^{2}\right)$ which sends $x \in S_{\omega}$ to $\left(f_{\omega}(x), \omega\right)$, what we just did was to put on $\mathcal{S} \rightarrow B\left(K_{S}^{2}\right)$ a structure of continuous family of compact genus $g$ Riemann surfaces for which $f$ is a topological trivialization. The map $f$ thus endows the family $\mathcal{S} \rightarrow B\left(K_{S}^{2}\right)$ with a Teichmüller structure. By the universal property of Teichmüller space, discussed in the preceding section, the map $\Phi$ is therefore continuous. We see that this rather simple proof of the continuity of $\Phi$ is a direct consequence of our definition of Teichmüller space in terms of Kuranishi families.

The uniqueness theorem of Teichmüller asserts that, among all admissible quasi-diffeomorphisms isotopic to it, $f_{\omega}: S \rightarrow S$ is one with minimal dilatation, and that it is uniquely characterized by this property.

Theorem 6.6 (Teichmüller's uniqueness theorem). Let $S$ be a genus $g$ Riemann surface with $g>1$, and let $\omega$ be a holomorphic quadratic differential on $S$. Assume that $\|\omega\|=k<1$. Let $f: S_{\omega} \rightarrow S$ be an admissible quasi-diffeomorphism which is isotopic to $f_{\omega}$ (i.e., isotopic to the identity). Then

$$
K[f] \geq K\left[f_{\omega}\right]=\frac{1+k}{1-k},
$$

and equality holds if and only if $f=f_{\omega}$.

We will not prove the theorem here, but we refer to one of the many proofs which exist in the literature (see, for example, [1,19], or [26] and the bibliography therein).

Assuming Teichmüller's uniqueness theorem, we shall first prove that the map $\Phi: B\left(K_{S}^{2}\right) \rightarrow T_{g}$ is injective. Suppose that $\Phi\left(\omega_{1}\right)=\Phi\left(\omega_{2}\right)$; in other words, that there are an isomorphism $\varphi: S_{\omega_{1}} \rightarrow S_{\omega_{2}}$ and an isotopy $f_{\omega_{1}} \sim f_{\omega_{2}} \circ \varphi$. Set $k_{i}=\left\|\omega_{i}\right\|, i=1,2$. The first part of Teichmüller's uniqueness theorem, together with Lemma 6.4, tells us that

$$
\frac{1+k_{2}}{1-k_{2}}=K\left[f_{\omega_{2}}\right]=K\left[f_{\omega_{2}} \circ \varphi\right] \geq K\left[f_{\omega_{1}}\right]=\frac{1+k_{1}}{1-k_{1}} .
$$


Reversing the roles of $\omega_{1}$ and $\omega_{2}$, we obtain $k_{1}=k_{2}$, and the second part of Teichmüller's uniqueness theorem implies that

$$
f_{\omega_{1}}=f_{\omega_{2}} \circ \varphi \text {. }
$$

Let $z_{i}$ be an $\omega_{i}$-coordinate on $S$, for $i=1,2$. By Lemma 6.4 we have

$$
k_{1} \frac{\partial}{\partial z_{1}} \otimes d \bar{z}_{1}=\mu_{f_{\omega_{1}}^{-1}}=\mu_{f_{\omega_{2}}^{-1}}=k_{2} \frac{\partial}{\partial z_{2}} \otimes d \bar{z}_{2} .
$$

But $k_{1}=k_{2}$, so that

$$
\overline{\frac{\partial z_{2}}{\partial z_{1}}}=\frac{\partial z_{2}}{\partial z_{1}} .
$$

Since $z_{2}$ is a holomorphic function of $z_{1}$, this implies that $z_{2}=c \cdot z_{1}+b$, where $b$ and $c$ are constants, and $c$ is real. Hence $\omega_{2}=\left(d z_{2}\right)^{2}=c^{2} \cdot\left(d z_{1}\right)^{2}=c^{2} \cdot \omega_{1}$. As $\left\|\omega_{1}\right\|=k_{1}=k_{2}=\left\|\omega_{2}\right\|$, we obtain that $c^{2}=1$, which proves the injectivity of $\Phi$.

We are now going to conclude the proof of Theorem 6.5, and hence of Theorem 6.1. The first step is the following.

Proposition 6.7. The Teichmüller map $\Phi$ is closed.

Proof. Let $\left\{\omega_{n}\right\}$ be a sequence in $B\left(K_{S}^{2}\right)$. Suppose the sequence $y_{n}=\Phi\left(\omega_{n}\right)$ converges to $y \in T_{g}$. What must be proved is that a subsequence of $\left\{\omega_{n}\right\}$ converges in $B\left(K_{S}^{2}\right)$. Set $y=[C,[f]]$, where $f$ is a diffeomorphism. As a neighborhood of $y$ we take the basis $B$ of a Kuranishi family $\pi: \mathcal{C} \rightarrow B$ for $C$. We can assume that there is a $C^{\infty}$ trivialization $(F, \pi): \mathcal{C} \rightarrow S \times B$ such that $F_{y}=f$. We may also assume that $\left\{y_{n}\right\} \subset B$. Set $f_{n}=F_{y_{n}}, \mu=\mu_{f}$ and $\mu_{n}=\mu_{f_{n}}$. Since $f$ and $f_{n}$ are $C^{\infty}$, the Beltrami differentials $\mu$ and $\mu_{n}$ are also $C^{\infty}$; since $F$ is $C^{\infty},\left\{\mu_{n}\right\}$ converges uniformly to $\mu$. Teichmüller's uniqueness theorem gives

$$
\frac{1+\|\mu\|}{1-\|\mu\|}=\lim _{n \rightarrow \infty}\left(\frac{1+\left\|\mu_{n}\right\|}{1-\left\|\mu_{n}\right\|}\right)=\lim _{n \rightarrow \infty} K\left[f_{n}\right] \geq \lim _{n \rightarrow \infty} K\left[f_{\omega_{n}}\right]=\lim _{n \rightarrow \infty}\left(\frac{1+\left\|\omega_{n}\right\|}{1-\left\|\omega_{n}\right\|}\right) .
$$

Therefore, for any constant $c$ with $\|\mu\|<c<1$, one has that $\left\|\omega_{n}\right\|<c$, if $n$ is large enough. Thus a subsequence of $\left\{\omega_{n}\right\}$ converges.

The last ingredient of the proof of Theorem 6.5 is the following.

Proposition 6.8. The Teichmüller space $T_{g}$ is connected.

We can immediately see that this implies Theorem 6.5. In fact, Proposition 6.7, in addition to showing that $\Phi\left(B\left(K_{S}^{2}\right)\right)$ is closed in $T_{g}$, also shows that $\Phi$ gives a homeomorphism between $B\left(K_{S}^{2}\right)$ and $\Phi\left(B\left(K_{S}^{2}\right)\right.$ ). But then the "invariance of domain" theorem says that $\Phi\left(B\left(K_{S}^{2}\right)\right)$ is open in $T_{g}$, since $B\left(K_{S}^{2}\right)$ and $T_{g}$ are differentiable manifolds of dimension $6 g-6$.

We now turn to Proposition 6.8. An immediate consequence of Theorem 6.2 and Lemma 6.3 is the following. 
Lemma 6.9. Let $\mu_{t}$ be a family of smooth Beltrami differentials on $S$, where $t$ varies in an interval $I \subset \mathbb{R}$. Suppose $\mu_{t}$ depends smoothly on $t$, in the sense that it is $C^{\infty}$ on $S \times I$. Then there are a differentiable family $\xi: Y \rightarrow I$ of Riemann surfaces and a differentiable trivialization $S \times I \rightarrow Y$ such that the Beltrami differential $\mu_{F_{t}}$ associated to $F_{t}$ is $\mu_{t}$.

To prove Proposition 6.8, denote by $x_{0}$ the base point [S, [1]] of $T_{S}=T_{g}$, let $x=[C,[f]]$ be another element of $T_{g}$, where $f: C \rightarrow S$ is a diffeomorphism, and set $\mu_{t}=t \mu_{f^{-1}}$. By Lemma 6.9, there is a differentiable family of curves with Teichmüller structure over an interval $I$ whose fiber at $t$ is $\left[Y_{t},\left[F_{t}^{-1}\right]\right]$. This comes from a differentiable map $\gamma: I \rightarrow T_{g}$, by the universal property of Teichmüller space. To prove connectedness, we just have to show that $\gamma(0)=x_{0}$ and $\gamma(1)=x$; in other words, that $\left[Y_{0},\left[F_{0}^{-1}\right]\right]=[S,[1]]$ and $\left[Y_{1},\left[F_{1}^{-1}\right]\right]=[C,[f]]$. Since, by construction, $\mu_{F_{0}^{-1}}=0$ and $\mu_{F_{1}^{-1}}=\mu_{f^{-1}}$, this follows from the second part of Lemma 6.4. This concludes the proof of Proposition 6.8, and therefore of Teichmüller's theorem.

\section{References}

[1] W. AвIKoff, "The Real Analytic Theory of Teichmüller Space", Lecture Notes in Mathematics, Vol. 820, Springer, Berlin, 1980.

[2] L. AHLFORS and L. BERS, Riemann's mapping theorem for variable metrics, Ann. of Math. (2) 72 (1960), 385-404.

[3] L. V. AhLFORS, The complex analytic structure of the space of closed Riemann surfaces., In: "Analytic Functions", Princeton Univ. Press, Princeton, N.J., 1960, 45-66.

[4] L. V. AHLFORS, Quasiconformal mappings, Teichmüller spaces, and Kleinian groups, In: “Fields Medallists' Lectures”, World Sci. Ser. 20th Century Math., Vol. 5, World Sci. Publ., River Edge, NJ, 1997, 10-23.

[5] L. V. AHLFORS, "Lectures on Quasiconformal Mappings", second ed., with supplemental chapters by C. J. Earle, I. Kra, M. Shishikura and J. H. Hubbard, University Lecture Series, Vol. 38, American Mathematical Society, Providence, RI, 2006.

[6] E. Arbarello, M. Cornalba, P. A. Griffiths and J. Harris, "Geometry of Algebraic Curves", Vol. II, Grundlehren der Mathematischen Wissenschaften, Vol. 268, Springer-Verlag, New York, 2009, to appear.

[7] L. BERS, "Riemann Surfaces", Notes by E. Rodlidtz and R. Pollack, Courant Institute of Mathematical Sciences, New York University, 1951-52.

[8] L. BERS, Quasiconformal mappings and Teichmüller's theorem, In: "Analytic Functions", Princeton Univ. Press, Princeton, NJ, 1960, 89-119.

[9] L. BERS, Spaces of Riemann surfaces, In: "Proc. Internat. Congress Math. 1958", Cambridge Univ. Press, New York, 1960, 349-361.

[10] L. BERs, Uniformization and moduli, In: "Contributions to Function Theory", Internat. Colloq. Function Theory, Bombay, 1960, Tata Institute of Fundamental Research, Bombay, 1960, 41-49.

[11] L. BERS, Uniformization by Beltrami equations, Comm. Pure Appl. Math. 14 (1961), 215228.

[12] L. BERS, "On Moduli of Riemann Surfaces", Mimeographed Lecture Notes, Eidgenössische Technische Hochschule, Zürich, 1964.

[13] L. Bers, On Teichmüller's proof of Teichmüller's theorem, J. Anal. Math. 46 (1986), $58-64$. 
[14] S.-SHEN CHERn, An elementary proof of the existence of isothermal parameters on a surface, Proc. Amer. Math. Soc. 6 (1955), 771-782.

[15] R. Courant and D. Hilbert, "Methods of Mathematical Physics: Partial Differential Equations", Vol. II by R. Courant., Interscience Publishers (a division of John Wiley \& Sons), New York-London, 1962.

[16] C. J. EARLE, Teichmüller spaces, Ann. Acad. Sci. Fenn. Math. 13 (1988), 355-361.

[17] C. J. EARle and J. Eells, JR., On the differential geometry of Teichmüller spaces, J. Anal. Math. 19 (1967), 35-52.

[18] D. B. A. Epstein, Curves on 2-manifolds and isotopies, Acta Math. 115 (1966), 83-107.

[19] F. P. GARDINER, "Teichmüller Theory and Quadratic Differentials", Wiley-Interscience Publication, Wiley, New York, 1987.

[20] A. GROTHENDIECK, "Techniques de construction en géométrie analytique. I. Description axiomatique de l'espace de Teichmüller et de ses variantes", In: "Familles d'Espaces Complexes et Fondements de la Géométrie Analytique", Séminaire Henri Cartan, 13ième année: 1960/61. Exposés No. 7 et 8, Sécretariat Mathématique, Paris, 1962.

[21] A. Grothendieck, "Techniques de construction en géométrie analytique. X. Construction de l'espace de Teichmüller", In: "Familles d'Espaces Complexes et Fondements de la Géométrie Analytique", Séminaire Henri Cartan, 13ième année: 1960/61, Exposé No. 17, Sécretariat Mathématique, Paris, 1962.

[22] Y. Imayoshi and M. TANiguchi, "An Introduction to Teichmüller Spaces", SpringerVerlag, Tokyo, 1992.

[23] L. KEEN, Intrinsic moduli on Riemann surfaces, Ann. of Math. (2) 84 (1966), 404-420.

[24] K. KodaIRA and D. C. SPENCER, On deformations of complex analytic structures. III. Stability theorems for complex structures, Ann. of Math. (2) 71 (1960), 43-76.

[25] A. KoRN, Zwei Anwendungen der Methode der sukzessiven Annäherungen, In: "Schwarz Festschrift", Berlin, 1914, 215-229.

[26] O. LeHTo, "Univalent Functions and Teichmüller Spaces", Graduate Texts in Mathematics, Vol. 109, Springer-Verlag, New York, 1987.

[27] L. LichtensteIn, Zur Theorie der konformen Abbildung nichtanalytischer, singularitätenfreier Flächenstücke auf ebene Gebiete, Bull. Int. de l'Acad. Sci. Cracovie, Sér. A (1916), 192-217.

[28] A. Marden and K. Strebel, A characterization of Teichmüller differentials, J. Differential Geom. 37 (1993), 1-29.

[29] C. B. MorRey, JR., On the solutions of quasi-linear elliptic partial differential equations, Trans. Amer. Math. Soc. 43 (1938), 126-166.

[30] E. REICH and K. STREBEL, Teichmüller mappings which keep the boundary point-wise fixed, In: "Advances in the Theory of Riemann Surfaces", Proc. Conf., Stony Brook, N.Y., 1969, Princeton Univ. Press, Princeton, N. J., 1971, 365-367.

[31] E. REICH and K. STREBEL, Extremal quasiconformal mappings with given boundary values, In: "Contributions to Analysis" (a collection of papers dedicated to Lipman Bers), Academic Press, New York, 1974, 375-391.

[32] M. SEPPÄLÄ and T. SORVALI, "Geometry of Riemann Surfaces and Teichmüller Spaces", North-Holland Mathematics Studies, Vol. 169, North-Holland Publishing Co., Amsterdam, 1992.

[33] E. Sernesi, "Deformations of Algebraic Schemes", Grundlehren der Mathematischen Wissenschaften, Vol. 334, Springer-Verlag, Berlin, 2006.

[34] K. Strebel, On the existence of extremal Teichmüller mappings, Complex Variables Theory Appl. 9 (1987), 287-295.

[35] O. TEICHMÜLLER, Extremale quasikonforme Abbildungen und quadratische Differentiale, Abh. Preuss. Akad. Wiss. Math.-Nat. Kl. 1939 (1940), no. 22, 197.

[36] O. TeIChmüller, Veränderliche Riemannsche Flächen, Deutsche Math. 7 (1944), 344-359. 
[37] O. TeICHMÜLLER, "Gesammelte Abhandlungen”, Springer-Verlag, Berlin, 1982, Edited and with a preface by Lars V. Ahlfors and Frederick W. Gehring.

[38] A. J. Tromba, "Teichmüller Theory in Riemannian Geometry", Lectures in Mathematics ETH Zürich, Birkhäuser Verlag, Basel, 1992, Lecture notes prepared by Jochen Denzler.

[39] J. H. HUBBARD, "Teichmüller Theory and Applications to geometry, Topology, and Dinamics", Vol. 1: "Teichmüller Theory". With contributions by A. Douady, W. Dunbar, R. Roeder, S. Bonnot, D. Brown, A. Hatcher, C. Hruska and S. Mitra, Matrix Editions, Ithaca, NY, 2006.

Dipartimento di Matematica

"G. Castelnuovo"

Sapienza Università di Roma

Piazzale A. Moro, 2

00185 Roma, Italia

ea@mat.uniroma1.it

Dipartimento di Matematica Università di Pavia

Via Ferrata, 1

27100 Pavia, Italia

maurizio.cornalba@unipv.it 